\title{
The asymptotic structure of counterflow diffusion flames for large activation energies
}

\author{
AMABLE LIÑ̃́N \\ Instituto Nacional de Técnica Aeroespacial and Escuela Técnica Superior de Ingenieros \\ Aeronáuticos, Madrid, Spain
}

(Received 7 September 1973)

\begin{abstract}
The structure of steady state diffusion flames is investigated by analyzing the mixing and chemical reaction of two opposed jets of fuel and oxidizer as a particular example. An Arrhenius onestep irreversible reaction has been considered in the realistic limit of large activation energies. The entire range of Damköhler numbers, or ratio of characteristic diffusion and chemical times, has been covered. When the resulting maximum temperature is plotted in terms of the Damköhler number (which is inversely proportional to the flow velocity) the characteristic $S$ curve emerges from the analysis, with segments from the curve resulting from:

(a) A nearly frozen ignition regime where the temperature and concentrations deviations from its frozen flow values are small. The lower branch and bend of the $S$ curve is covered by this regime.

(b) A partial burning regime, where both reactants cross the reaction zone toward regions of frozen flow. This regime is unstable.

(c) A premixed flame regime where only one of the reactants leaks through the reaction zone, which then separates a region of frozen flow from a region of near-equilibrium.

(d) A near-equilibrium diffusion controlled regime, covering the upper branch of the $S$ curve, with a thin reaction zone separating two regions of equilibrium flow.
\end{abstract}

Analytical expressions are obtained, in particular, for the ignition and extinction conditions.

\section{Introduction}

Two basic classes of non premixed combustion problems can be defined as "initial-value problems", described either by ordinary differential equations with boundary conditions at only one point or by parabolic partial differential equations, and "boundary-value problems", described by ordinary differential equations with two boundary points or by elliptic partial differential equations. From a physical point of view, solutions to initial-value problems are unique, but multiple solutions to boundary-value problems may exist corresponding to drastically different combustion regimes, this being especially true for large activation energies.

Stagnation-point counterflow of fuel and oxidizer [1-7], combustion of a spherical fuel droplet in an oxidizing atmosphere [8-12], planar one dimensional interdiffusion and reaction of fuel and oxidizer [14-16], are among the boundary-value problems that have been studied in efforts to clarify diffusion-flame structure. We shall carry out in this paper an asymptotic analysis for large activation energies of diffusion-flame structure in problems of the boundary-value type. Since the qualitative aspects of flame structure are independent of the specific choice of problems within the boundary-value class, we have selected the counter-flow diffusion flame as a model. 
In unpremixed combustion, the maximum temperature in the flow field depends on a Damköhler number, defined as the ratio of a diffusion time to a reaction time evaluated at a boundary temperature. For low activation energies the dependence is represented by a monotonic function which goes from the highest boundary temperature at zero Damköhler number to an adiabatic flame temperature at infinite Damköhler number. For high activation energies and adiabatic flame temperatures sufficiently higher than the boundary temperatures, the curve develops an $S$ shape, with the ignition conditions clearly defined as the position of the low-temperature vertical tangent and the extinction conditions by the high temperature vertical tangent. That only the portion of the curve between the vertical tangents is dynamically unstable has been demonstrated by transient stability analysis, under suitably restricted conditions [16].

The curve giving the maximum temperature vs the Damköhler number has been generated by somewhat laborious numerical solutions of the steady state equations $[3-8,15,16]$ and by approximate methods of an ad hoc nature [1, 8-10]. Asymptotic methods were first used in the form of boundary layer type analysis of non-equilibrium effects in the limit of large Damköhler numbers [2, 14]. Later singular perturbation methods were used $[3,11,12]$ to refine the asymptotic solution by retaining higher order terms in an expansion for large Damköhler numbers. A regular expansion for low Damköhler numbers was also carried out [3] for the stagnation point diffusion flame. Asymptotic methods that treat the Damköhler number as the large or small parameter are incapable of defining ignition or extinction conditions so that wide use has been made of numerical methods for this purpose.

Adoption of the activation energy, or more specifically the ratio of activation temperature to the characteristic temperature of the system, as the large parameter in the present work produces analytical results for the entire range of the Damköhler number, readily exhibits the various parametric dependences, accurately covers conditions of greatest practical interest and generally provides a much clearer physical understanding of the process involved.

In Section 2 we formulate the problem and identify the limiting solutions and combustion regimes. In Sections 3 to 6 we consider in detail the ignition, partial burning, premixed flame and diffusion flame regimes. In Section 7 we summarize the results and compare with other numerical results.

Initial value problems in combustion have been analyzed for large activation energies by Liñán and Crespo[25].

\section{Formulation}

We shall analyze the simultaneous mixing and chemical reaction at the stagnation point of two counter-flow streams of fuel and oxidizer. A one-step irreversible Arrhenius reaction will be considered, which is first order with respect to both fuel and oxidizer.

Even though the asymptotic analysis may be performed easily for more complex fluid dynamic systems, we shall assume, for simplicity in the presentation, that the two streams have equal velocity and that the density, specific heat, and 
transport coefficients are constant. We shall use Fick's law, with equal diffusivities of mass and heat, to calculate the diffusion velocities. The SchvabZeldovich procedure then leads to the following equation for describing the temperature field (see Ref. [3] or [4] for the details of the derivation):

$$
\frac{d^{2} T}{d z^{2}}+z \frac{d T}{d z}=-D y_{0} y_{F} \exp \left(-T_{a} / T\right)
$$

with the boundary conditions

$$
T=T_{\infty} \quad \text { at } \quad z \rightarrow \infty \quad \text { and } \quad T=T_{-\infty} \quad \text { at } \quad z \rightarrow-\infty
$$

Here $T$ is the temperature made nondimensional with the characteristic temperature $Q / c_{p} Y_{F-\infty}$, obtained from the heat release per unit mass of fuel $Q$, the specific heat $c_{p}$ and the fuel mass fraction in the fuel stream. The distance normal to the mixing layer $z$, measured from the plane of the stagnation point has been made nondimensional with the characteristic mixing length $\sqrt{D_{i f} / A}$ where $D_{\text {if }}$ is the diffusion coefficient and $A$ is the stagnation point velocity gradient, or ratio between velocity and distance normal to the mixing layer. The fuel stream approaches from $z=-\infty$, and the oxidizer stream from $z=+\infty$ where the oxidizer mass fraction is $Y_{0 \times \infty}$. The Damköhler number $D=Z \nu Y_{F-\infty} / A$ is based on the frequency factor $Z$ of the reaction rate and the characteristic flow time $A^{-1}$ of the velocity field, with the stoichiometric mass ratio $\nu$ of oxidizer to fuel appearing as a factor. $T_{a}$ is the nondimensional activation energy. The factors $y_{0}$ and $y_{F}$ appearing in (1) are the oxidizer and fuel mass fractions divided by $\nu Y_{F-\infty}$ and $Y_{F-\infty}$ respectively. They are given by the relations

with

$$
\begin{aligned}
& y_{0}=\alpha(1-x)+T_{f}-T \\
& y_{F}=x+T_{f}-T
\end{aligned}
$$

$$
T_{f}=T_{\infty}-\beta x
$$

where

$$
x=(1 / 2) \operatorname{erfc}(z / \sqrt{2})
$$

$T_{f}$ is the nondimensional frozen flow temperature with $\beta=\left(T_{\infty}-T_{-\infty}\right)$ which we assume, without loss of generality, to be larger than zero. The normalized oxidizer mass fraction at the oxidizer stream is $\alpha=Y_{0 \infty} / \nu Y_{F-\infty}$.

Equations (1) and (2) when written in terms of $x$ take the following form

$$
\frac{d^{2} T}{d x^{2}}=-2 \pi \exp \left(z^{2}\right) D y_{0} y_{F} \exp \left(-T_{a} / T\right)
$$

where the factor $2 \pi \exp \left(z^{2}\right)=(d z / d x)^{2}$ should be written in terms of $x$ by using (5); its dependence on $x$ results from the convective term in Eq. (1).

The boundary conditions are

$$
T=T_{\infty} \quad \text { at } \quad x=0 \quad \text { and } \quad T=T_{\infty}-\beta \quad \text { at } \quad x=1
$$

Then, the normalized fuel and oxidizer concentrations are, respectively, 1 and 0 at $x=1$, and 0 and $\alpha$ at $x=0$. 
Our purpose is to obtain the asymptotic solution of (6) and (7) for large values of $T_{a}$, with $\alpha, \beta, T_{\infty}$ of order unity and the Damköhler number $D$ ranging from zero to infinity.

If in Eq. (6) we take the limit $T_{a} \rightarrow \infty$ and consider $D$ to be a function of $T_{a}$, the following limiting equations are obtained depending on the resulting local value of $T$ and our choice of $D\left(T_{a}\right)$ :

(a) Frozen flow with

$$
d^{2} T / d x^{2}=0
$$

Therefore $T$ is a linear function of $x$.

(b) Equilibrium flow with zero oxidizer concentration

$$
y_{0}=0 \rightarrow T=\alpha(1-x)+T_{\infty}-\beta x
$$

(c) Equilibrium flow with zero fuel concentration

$$
y_{F}=0 \rightarrow T=x+T_{\infty}-\beta x
$$

The four different possible regimes that we shall encounter for large $T_{a}$ show the existence within the flow field of two regions, where the temperature is given in the first approximation by Eqs. (8) to (10), separated by a thin reaction zone. The reaction zone is infinitely thin in the limit $T_{a} \rightarrow \infty . \dagger$

The flame temperature is a possibly multivalued function of the Damköhler number, so that ignition-extinction condition will be exhibited. When the flame temperature varies from the boundary temperature $T_{\infty}$ to the adiabatic flame temperature we may encounter a nearly frozen ignition regime, a partial burning regime, a pre-mixed flame regime and finally a diffusion controlled or diffusion flame regime. We shall describe below these combustion regimes. For the temperature distributions, $T$ as a function of $x$, corresponding to these possible regimes, the reader should refer to Fig. 1 for the cases where $\beta>1 / 2$, to Fig. 2 for $\beta<1 / 2$ and $\alpha+2 \beta>1$, and to Fig. 3 for $\beta<1 / 2$ and $\alpha+2 \beta<1$. The temperature distribution corresponding to the diffusion flame regime, for example, is given by two segments of the lines $y_{0}=0$ and $y_{F}=0$. The temperature distributions have been shown for frozen flow (F.F.), and for two examples of the partial burning (P.B.) and premixed flame (P.F.) regimes. The thick dashed line in these figures gives the possible flame temperature and the flame location for all Damköhler number ranging from zero to infinity.

1. Frozen flow-Ignition regime. In this regime the chemical reaction is frozen in the first approximation and the temperature is given by

$$
T=T_{f}=T_{\infty}-\beta x
$$

which satisfies the boundary conditions (7). The oxidizer and fuel concentrations

†Libby and Economos [24] introduced a distributed flame-zone model of combustion in a nonpremixed system, in which the reaction was treated as being frozen below an artificially introduced temperature. This model is not applicable to the present kinetic scheme. 


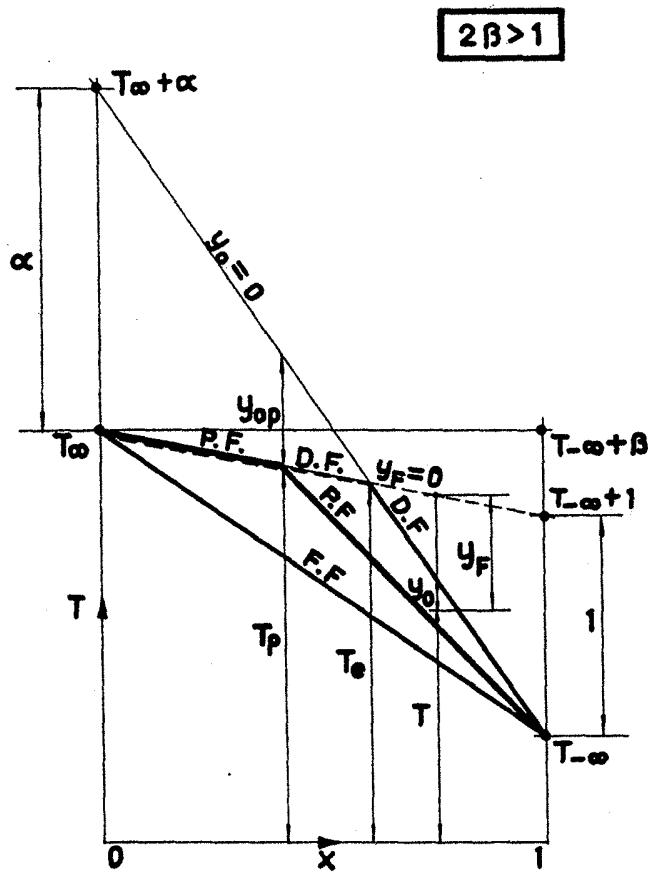

Fig. 1. Asymptotic temperature and concentration distributions for the frozen flow (F.F.), premixed flame (P.F.), and diffusion controlled (D.F.) regimes in terms of the strained coordinate $x$. No partial burning regime exists in this case, where $T_{e}<T_{\infty}$.

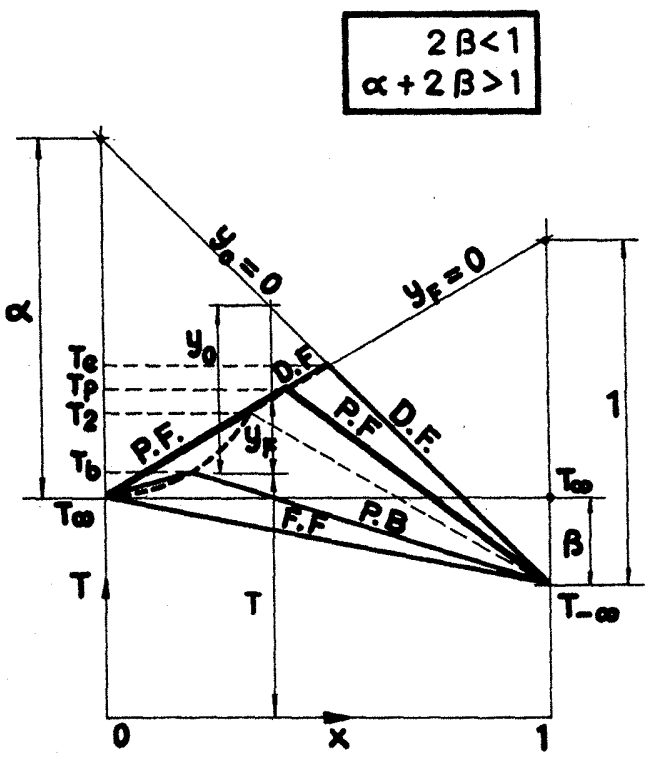

Fig. 2. Asymptotic temperature and concentration distributions for the frozen flow, partial burning (P.B.), premixed flame, and diffusion controlled regime for the case $2 \beta<1$ and $\alpha+2 \beta>1$, when the flame lies always in the lean side of the mixing layer. 


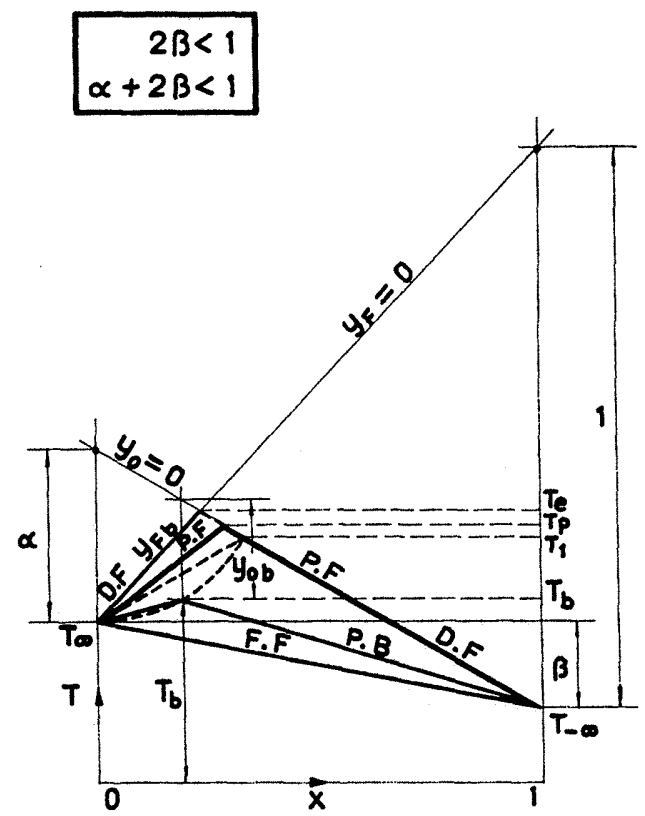

Fig. 3. Asymptotic temperature and concentration distributions for the case when $\alpha+$ $2 \beta<1$. In the premixed flame regime the flame lies on the rich side of the mixing layer.

are then given by

$$
y_{o f}=\alpha(1-x) \quad \text { and } \quad y_{F f}=x
$$

To obtain the temperature in the second approximation, in an expansion for large $T_{\infty}{ }^{2} / T_{a}$, chemical reaction effects must be retained. For a sufficiently large Damköhler number the temperature rise above the frozen flow value is of order $T_{\infty}{ }^{2} / T_{a}$, so that the nonlinear effects associated with the Arrhenius exponent are important. These effects are responsible for a rapid increase in the product concentration for large Damköhler numbers when $\beta>1$, and for the existence, when $\beta<1$ of two solutions if $D$ is lower than an "ignition Damköhler number" and no solutions above this value. Hence the lower bend of the $S$ curve is described by the ignition regime analysis. The condition $\beta<1$ is equivalent to the statement that the boundary temperature $T_{\infty}$ is lower than the adiabatic flame temperature $T_{e}=T_{\infty}+(1-\beta) \alpha /(1+\alpha)$.

2. Partial burning regime. In this regime two frozen flow regions are separated by a thin, infinitely thin in the limit $T_{a} \rightarrow \infty$, reaction region which acts as sink for the reactants; because the reaction rate is not sufficiently fast, leakage of both reactants through the flame occurs.

In the first approximation, the temperature is given by

$$
T=T_{\infty}+\left(T_{b}-T_{\infty}\right)\left(x / x_{b}\right), \quad \text { for } \quad x<x_{b}
$$

and

$$
T=T_{\infty}-\beta+\left(T_{b}-T_{\infty}+\beta\right)(1-x) /\left(1-x_{b}\right) \quad \text { for } \quad x>x_{b}
$$


The flame is located at $x=x_{b}$ where the temperature is $T_{b}$ and the fuel and oxidizer concentration $y_{F b}$ and $y_{o b}$ as given by (3) and (4).

As we shall show below, the matching conditions with the asymptotic solution describing the temperature field within the thin reaction zone (a) indicate that the temperature distribution, obtained from (13) and (14), in the outer frozen flow regions should decrease at the same rate on both sides of the flame and (b) provide a relation between $T_{b}$ and the Damköhler number $D$. From the equality of the slopes of the temperature distribution on both sides of the flame we deduce

$$
x_{b}=\left\{2+\beta /\left(T_{b}-T_{x}\right)\right\}^{-1}
$$

This regime is only possible for $\beta<1 / 2$. The flame temperature $T_{b}$ is lower than $T_{\infty}-\beta+1 / 2$ when $\alpha+2 \beta>1$, otherwise $y_{F b}$ would become negative. When $\alpha+2 \beta<1, T_{b}$ is limited by $T_{\infty}+\alpha / 2$ so as to have $y_{o b}>0$.

3. Premixed flame regime. In this regime a frozen flow region, and a equilibrium region are separated by a thin reaction region with leakage of only one of the reactants through the flame. The structure of the reaction zone is the same as the structure of the reaction zone of a premixed flame with heat loss toward the equilibrium side of the flame. Two possibilities arise depending on the value of $\alpha+2 \beta$.

For $\alpha+2 \beta>1$, we find equilibrium flow with $y_{F}=0$ for $x<x_{\mathrm{p}}$

$$
T=T_{\infty}+(1-\beta) x
$$

and frozen flow for $x>x_{p}$, with

$$
T=T_{\infty}-\beta+\left(T_{p}-T_{\infty}+\beta\right)(1-x) /\left(1-x_{p}\right)
$$

Here $T_{p}$ is the value of $T$ at $x=x_{p}$ as obtained from (16). At the flame $y_{0}=y_{o p}=\alpha-(\alpha+1) x_{p}$.

For $\alpha+2 \beta<1$, equilibrium, with $y_{0}=0$, occurs for $x>x_{p}$, with $T$ given by

$$
T=T_{\infty}+\alpha-(\alpha+\beta) x
$$

and frozen flow for $x<x_{p}$, with

$$
T=T_{\infty}+\left(T_{p}-T_{\infty}\right)\left(x / x_{p}\right)
$$

In this case at $x=x_{p}, y_{F}=y_{F_{p}}=-\alpha+(\alpha+1) x_{p}$.

The two alternatives result from the fact that matching with an inner reaction zone solution, with complete burning of one reactant in the first approximation, is possible only if the temperature decays toward the equilibrium side of the flame at a slower rate than toward the frozen flow side. The matching conditions, in addition, will provide a relation between $T_{p}$ and $D$.

4. Diffusion flame or near-equilibrium regime. For sufficiently high Damköhler numbers a diffusion controlled regime exists in which equilibrium is reached, to the first approximation, on both sides of a thin reaction zone. Then,

$$
\begin{array}{lll}
x<x_{e} & : & Y_{F}=0 \rightarrow T=T_{\infty}+(1-\beta) x \\
x>x_{e} & : & Y_{0}=0 \rightarrow T=T_{\infty}+\alpha-(\alpha+\beta) x .
\end{array}
$$


At the flame the temperature takes the adiabatic flame value

$$
T_{e}=T_{\infty}+(1-\beta) \alpha /(1+\alpha)
$$

and the flame is located at $x_{e}$,

$$
x_{e}=\alpha /(\alpha+1)
$$

We shall see below, when analyzing the structure of the thin reaction zone, that for $\beta<1$ and sufficiently high activation energies multiple solutions may exist for Damköhler numbers above a minimum "extinction $D$ " and no solution for $D$ below this value.

There are cases in which the extinction conditions do not occur under this regime but under the previous premixed flame regime. This is the case, for example, if $(1-\beta)$ is small compared with unity.

In all these regimes the asymptotic flame structure is determined, in the first approximation, independently of chemical kinetics, with the exception of the partial burning and premixed flame regimes in which an unknown flame temperature, $T_{b}$ or $T_{p}$, appears which will be a function of the Damköhler number. The relation between flame temperature and Damköhler number will be obtained, sequentially for the different regimes in the following sections, from the matching conditions between the asymptotic expansions for the temperature in the reaction zone and in the outer regions.

\section{Nearly frozen, ignition, regime}

As indicated before, in this regime the Damköhler number is such that the temperature rise, above the frozen flow value, due to the chemical production term, is of order $T_{\infty}{ }^{2} / T_{a}$, which is small compared with $T_{\infty}$, but large enough so as to force us to retain in the equations the nonlinear effects associated with the Arrhenius exponent. These effects are responsible for the ignition characteristics.

We shall use $\epsilon=T_{\infty}{ }^{2} / T_{a}$ as the small parameter, and begin by analysing the case where the temperature difference $\beta$ between the two streams is of order $\epsilon$. We look for the Damköhler number $D$ that produces, at $x=1 / 2$ for example, a given increment in temperature of order $\epsilon$ above the frozen flow value. We choose $D$ as the dependent variable because $D$ is a single valued function of that temperature increment while the inverse function may be multivalued.

We shall introduce in Eq. (6) the expansions

$$
\begin{gathered}
T=T_{\infty}+\epsilon\left(\theta_{1}-\beta_{1} x\right)+\epsilon^{2} \theta_{2}+\cdots \\
D \alpha\left(T_{a} / T_{\infty}{ }^{2}\right) \exp \left(-T_{a} / T_{\infty}\right)=\Delta_{0}+\epsilon \Delta_{1}+\cdots
\end{gathered}
$$

where $\theta_{1}(x), \ldots, \Delta_{0}, \ldots$, which we anticipate to be of order unity, are to be determined in terms of $\theta_{1}(1 / 2)$, with $\theta_{2}(1 / 2)=\theta_{3}(1 / 2)=0$, or chosen for convenience; we wrote $\beta_{1}=\beta / \epsilon$. The following equation is obtained for $\theta_{1}$

$$
\frac{d^{2} \theta_{1}}{d x^{2}}=-2 \pi \exp \left(z^{2}\right) \Delta_{0}(1-x) x \exp \left(\theta_{1}-\beta_{1} x\right)
$$

to be solved with the boundary conditions $\theta_{1}(0)=\theta_{1}(1)=0$. 
The value of $\theta_{1}(1 / 2)$ obtained from Eq. (26) depends on the reduced Damköhler number $\Delta_{0}$, or vice versa. Equation (26) may also be obtained from Eq. (6) by neglecting the reactant consumption and linearizing the Arrhenius exponent around the higher boundary temperature in the manner of FrankKamenetskii [17]. There are no solutions of this equation for $\Delta_{0}$ larger than a critical "ignition" value $\Delta_{0 I}$ which is a function of $\dot{\beta}_{1}$, and there are two solutions for $\Delta_{0}<\Delta_{01}$. Of course if we specify $\theta_{1}(1 / 2)$ we then obtain a unique $\Delta_{0}$ lower than $\Delta_{01}$.

Figure 4 shows $\theta(x)$ for $\beta_{1}=0$ and several values of $\Delta_{0}$ as obtained from a
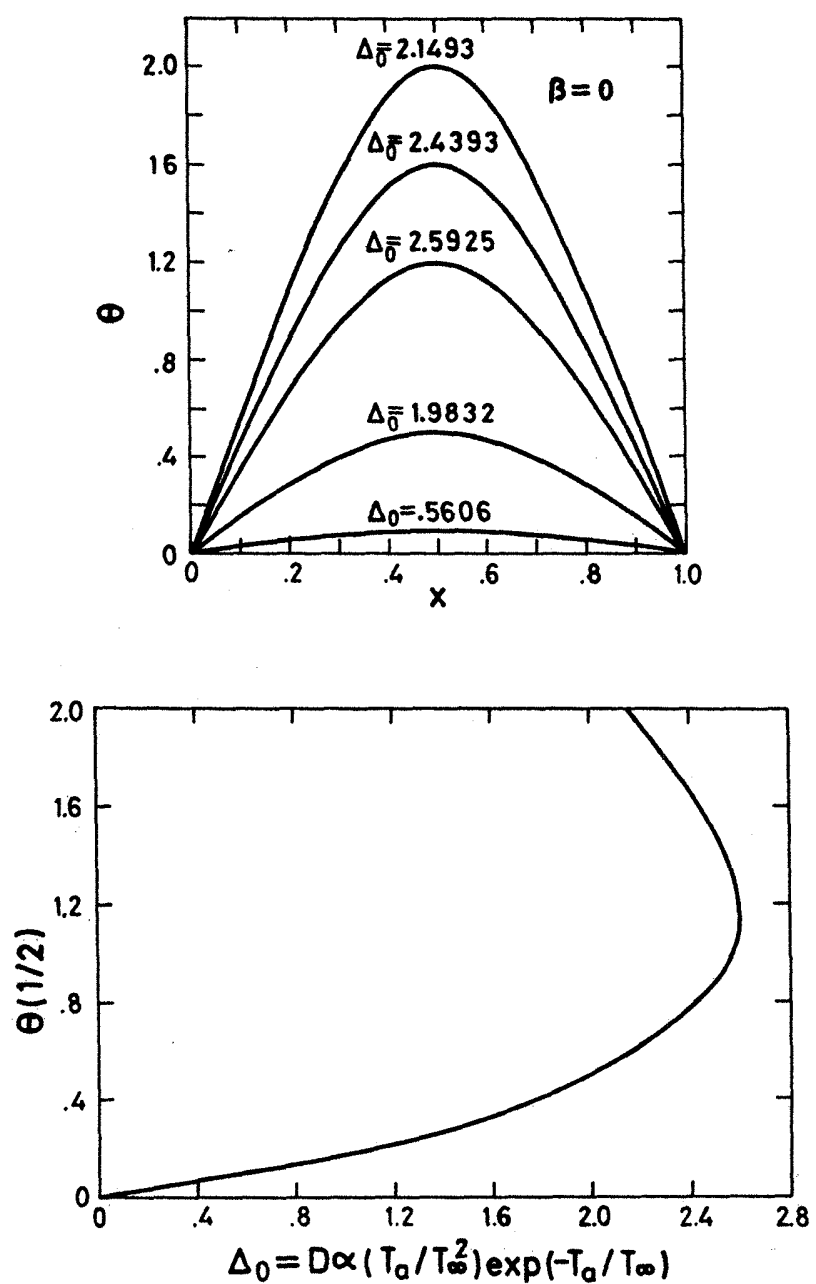

Fig. 4. Temperature distribution in the nearly frozen regime, for $\beta=0$ and several values of the reduced Damköhler number $\Delta_{0}$. The lower part of the figure shows the maximum temperature as a double-valued function of the Damköhler number for $\Delta_{0}<$ $\Delta_{0 I}=2.5925$. 
numerical solution. Also shown in Fig. 4 is $\theta_{1}(1 / 2)$, the maximum temperature in this case, as a function of $\Delta_{0}$; the lower bend of the $S$ curve is thus reproduced.

A first approximation for the Damköhler number of ignition, obtained from Eq. (25), is given by

$$
\epsilon^{-1} D_{l} \alpha \exp \left(-T_{a} / T_{\infty}\right)=\Delta_{0 I}(\beta / \epsilon) .
$$

The reduced Damköhler number of ignition $\Delta_{0 I}\left(\beta_{1}\right)$, represented in Fig. 5, increases with increasing $\beta_{1}$.

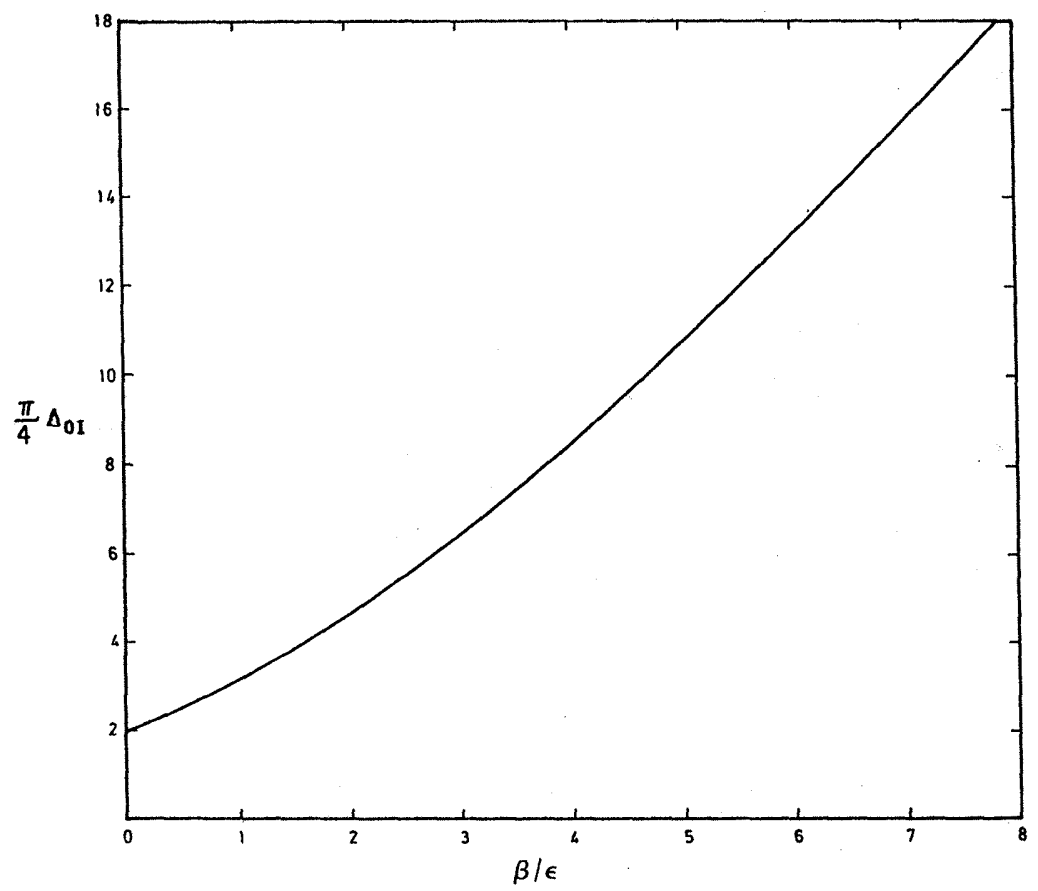

Fig. 5. The reduced Damköhler number of ignition $\Delta_{0 r}$ in terms of $\beta / \epsilon$.

For large values of $\beta_{1}$, the chemical reaction, is limited to a region near the hot boundary such that $\beta_{1} x$ is of order unity; for larger values of $x$ the Arrhenius factor becomes exponentially small. The analysis of the nearly frozen regime for large values of $\beta_{1}$, or $\beta$ of order unity, is left for the Appendix A. There we obtain the following asymptotic expression for the Ignition Damköhler number

$$
D_{\mathrm{l}} \alpha \exp \left(-T_{a} / T_{\infty}\right)=2 e^{-2}(1-\beta)^{-2} F(\beta) \ln \left\{\beta^{2}(1-\beta)^{2} T_{a}^{2} / 8 \pi T_{\infty}^{4}\right\},
$$

where $F(\beta)$ is very accurately correlated by $F(\beta)=2 \beta-\beta^{2}$.

Multiple solutions, and thereby clearly defined ignition conditions, are obtained only for $\beta<1$.

For $\beta$ close to one, in the ignition conditions the reaction zone structure is of the same form as that of the premixed flame regime, and then the results obtained in Section 5 may also be used to determine the ignition conditions. For small 
values of $\beta$, although large $\beta_{1}$, the Damköhler number of ignition can be determined by both Eqs. (27) and (28).

\section{Partial burning regime}

In this regime, which is only possible for $\beta<1 / 2$, two regions of frozen flow are separated by a thin reaction region. If the activation temperature is large compared with the local temperature a very small change in this temperature below the maximum is necessary to freeze the chemical reaction. Both reactants leak through the reaction zone where they undergo a very small relative change in concentration.

The Damköhler number is large enough to make the reaction time at the maximum temperature of the same order as the diffusion time through the reaction zone. In the frozen flow regions the temperature, which, for large $T_{a}$, is given in the first approximation by Eqs. (13) and (14), being smaller than the maximum temperature, $T_{b}$ in the first approximation, produces a chemical reaction time exponentially large in $T_{a} / T$ compared with the diffusion time. Only at both outer edges of the mixing layer will, again, the large reaction time be comparable with an appropriately large residence time in thick zones, where the chemical reaction will go to completion without a significant increase in temperature. The existence of these thick reaction zones at the outer edges does not affect, to any algebraic order, the solution in the frozen flow regions or in the interior reaction zones and therefore we shall not analyze it here.

Peskin and Wise presented in Ref.[9] a method for the analysis of nonequilibrium diffusion flames in which they assume the reaction zone to be infinitely thin allowing both reactants to leak through the flame. However they chose the flame location and the amount of reactants burning in the thin flame artificially, instead of basing this choice on the matching conditions with an asymptotic reaction zone solution for large activation energies.

We shall use $\epsilon=T_{b}^{2} / T_{a}$, as the small parameter, and pose our problem as to find the Damköhler number and the asymptotic solution for small $\epsilon$ of Eqs. (6) and (7), when the temperature in the frozen region to the right of the reaction zone, $\left(x-x_{b}\right) / \epsilon \gg 1$, is given to all algebraic orders in $\epsilon$, by Eq. (14), with $x_{b}$ given in terms of $T_{b}$ by Eq. (15).

In the frozen flow region toward the oxidizer side, $\left(x_{b}-x\right) / \epsilon \gg 1$, the chemical reaction will also be frozen to all algebraic orders in $\epsilon$. However, the temperature will be given by Eq. (13) only in the first approximation, as we shall see below. We use the following outer expansion for this region,

$$
T=T_{\infty}+\left(T_{b}-T_{\infty}\right)\left(x / x_{b}\right)+\epsilon B_{1} x+\epsilon^{2} B_{2} x+\cdots
$$

which reflects the fact, that the chemical reaction is frozen to all algebraic orders in $\epsilon$.

For the temperature within the inner reaction zone we use the expansion

$$
T=T_{b}+\epsilon \Phi_{1}(\zeta)+\epsilon^{2} \Phi_{2}(\zeta)+\cdots
$$

in terms of the inner variable

$$
\zeta=\left(x-x_{b}\right) / \epsilon
$$


We also expand $D$ as follows

$$
D=D_{b}\left(1+b_{1} \epsilon+b_{2} \epsilon^{2}+\cdots\right)
$$

where $D_{b}$ will be the Damköhler number which makes matching possible, to the first approximation, with the outer solutions (13) and (14). If (30) and (32) are introduced in Eq. (6), previously written in terms of $\zeta$, we obtain the following equation for $\Phi_{1}$, free from convective effects,

where

$$
d^{2} \Phi_{1} / d \zeta^{2}=-\Lambda_{0} \exp \Phi_{1}
$$

$$
\begin{aligned}
& \Lambda_{0}=\left(T_{b}^{2} / T_{a}\right) 2 \pi \exp \left(z_{b}^{2}\right) D_{b} y_{0 b} y_{F b} \exp \left(-T_{a} / T_{b}\right) \\
& y_{0 b}=\alpha+T_{\infty}-T_{b}-(\alpha+\beta)\left\{2+\beta /\left(T_{b}-T_{\infty}\right)\right\}^{-1} \\
& y_{F b}=T_{\infty}-T_{b}+(1-\beta)\left\{2+\beta /\left(T_{b}-T_{\infty}\right)\right\}^{-1}
\end{aligned}
$$

are the oxidizer and fuel concentration in the reaction zone in the first approximation; $z_{b}$ is the value of $z$, as given by Eq. (5), at $x=x_{b}$. From Eq. (33) we obtain

$$
\left(d \Phi_{1} / d \zeta\right)^{2}=2 \Lambda_{0}\left(\exp \Phi_{m}-\exp \Phi_{1}\right)
$$

where $\Phi_{m}$ is the maximum value of $\Phi_{1}$. From Eq. (34) we obtain

$$
2 \ln \left(t\left\{1+\sqrt{1-1 / t^{2}}\right\}\right)= \pm\left(2 \Lambda_{0} \exp \Phi_{m}\right)^{1 / 2}\left(\zeta-\zeta_{m}\right)
$$

choosing the plus and minus sign, respectively, for $\left(\zeta-\zeta_{m}\right)$ positive and negative. The constant of integration $\zeta_{m}$ gives the location in $\zeta$ of the maximum temperature. In this equation $t=\exp \left\{\left(\Phi_{m}-\Phi_{1}\right) / 2\right\}$.

We must determine $\zeta_{m}$, together with $\Phi_{m}$ and the reduced Damköhler number $\Lambda_{0}$, from the matching conditions between the inner expansion, Eq. (30), and the outer expansions, Eqs. (14) and (29).

Matching to zero order in $\epsilon$ is automatic because we anticipated that the temperature given by the outer expansions, in the first approximation, would be equal to $T_{b}$, which was also chosen as the first term of the inner expansion.

Matching to the next order, intermediate between $\epsilon^{0}$ and $\epsilon$, carried out in intermediate variables, demands the equality, at the outer edges of the reaction zone, of the slopes of the temperature distribution given by the inner and outer expansions. Then we obtain,

$$
\begin{aligned}
\left(2 \Lambda_{0} \exp \Phi_{m}\right)^{1 / 2} & =\left(T_{b}-T_{\infty}\right) / x_{b} \\
\left(T_{b}-T_{\infty}\right) / x_{b} & =\left(T_{b}-T_{\infty}+\beta\right) /\left(1-x_{b}\right)
\end{aligned}
$$

This last relation (37) states that in the outer frozen flow region the temperature, in the first approximation, decreases with the same rate on both sides of the flame, as anticipated in Section 2, where Eq. (37) is written as Eq. (15).

Matching terms of order $\epsilon$ yields

$$
\begin{aligned}
& \Phi_{m}+\ln 4+\zeta_{m}\left(T_{b}-T_{\infty}\right) / x_{b}=0 \\
& \Phi_{m}+\ln 4-\zeta_{m}\left(T_{b}-T_{\infty}\right) / x_{b}=B_{1} x_{b}
\end{aligned}
$$

Eqs. (36), (38) and (39) are not sufficient to determine $\Lambda_{0}, \Phi_{m}, \zeta_{m}$ and $B_{1}$. The new 
relation must be obtained from the matching conditions with the three term expansion of the inner solution. The details are left for the Appendix B where we obtain

$$
B_{1}=2\left\{(\alpha+\beta) / y_{0 b}+(\beta-1) / y_{F b}-4 \pi z_{b} \exp \left(z_{b}^{2}\right)\right\}
$$

Equations (36) to (40) may be used to determine, in terms of $T_{b}$ for example, the maximum temperature $T_{b}+\epsilon \Phi_{m}$, and its location $x_{b}+\epsilon \zeta_{m}$, in the second approximation. We may simultaneously obtain $B_{1}$, which gives the temperature in the frozen flow region $x<x_{p}$, and $D_{b}$, the first approximation for the Damköhler number. Explicitly,

$$
D_{b} y_{0 b} y_{F b} \exp \left(-T_{a} / T_{b}\right)=\left(T_{a} / \pi\right)\left(T_{b}-T_{\infty}\right)^{2}\left(T_{b} x_{b}\right)^{-2} \exp \left(-z_{b}^{2}+\Phi_{m}\right)
$$

where

$$
\Phi_{m}=-\ln 4+x_{b}\left\{(\alpha+\beta) / y_{0 b}+(\beta-1) / y_{F b}-4 \pi z_{b} \exp z_{b}^{2}\right\}
$$

The range of flame temperatures in this partial burning regime is limited by the condition that $y_{0 b}$ and $y_{F b}$ must be larger than zero. The upper limiting values of $T_{b}$ are, therefore, $T_{1}=T_{\infty}+\alpha / 2$ for $\alpha+2 \beta<1$ when $y_{0 b}$ becomes zero, and $T_{2}=$ $T_{\infty}-\beta+1 / 2$ for the case $\alpha+2 \beta>1$ when $y_{F b}$ becomes zero.

The flame temperature, $T_{b}$, vs Damköhler number, $D_{b}$, resulting from (41) will have an $S$ shaped form, exhibiting thereby, ignition extinction characteristics.

However, of the three branches of the $T_{b}\left(D_{b}\right)$ curve, the lower, the ignition branch, will correspond to small values of $T_{b}-T_{\infty}$ that are not large compared with $T_{\infty}{ }^{2} / T_{a}$; so that the analysis given in this section is not valid for this branch, because the thickness of the reaction zone becomes of order unity if $\beta \sim T_{\infty}{ }^{2} / T_{a}$, or of order $x_{b}$ if $\beta \gg T_{\infty}{ }^{2} / T_{a}$. Thus the analysis given in the previous section must be used to describe the ignition branch.

The middle branch, where $T_{b}$ increases with decreasing $D_{b}$, will very likely turn out to be unstable in a stability analysis similar to that presented by Kirkby and Schmitz $[15,16]$.

A third upper branch of the curve $T_{b}\left(D_{b}\right)$ exists because $D_{b}$ goes to infinity when $y_{0 b}$ or $y_{F b}$ go to zero; in this case, the expansion for the reaction zone will fail, and the premixed flame analysis of the following section should be used. Only in the particular case $\alpha+2 \beta=1$, for which $y_{0 b}$ and $y_{F b}$ go to zero simultaneously, will the diffusion controlled regime appear sequentially to the partial burning regime when $T_{b}$ approaches $T_{e}$. The extinction conditions cannot be determined from the analysis of this regime.

\section{Premixed flame regime}

While in the previous regime leakage of both reactants through the flame occurred, in this premixed flame regime the flame acts, in the first approximation for large $T_{a}$, as a complete sink for one of the reactants, the fuel if $\alpha+2 \beta>1$, and the oxidizer if $\alpha+2 \beta<1$. In this approximation the temperature is given by Eqs. (16) and (17) or (18) and (19), the results corresponding to frozen flow on one side of the flame and equilibrium flow on the other.

If $\beta>1$, the temperature in the reaction zone, which is close to $T_{p}$, will be lower than the temperature in the equilibrium region, which ranges from $T_{p}$ to $T_{\infty}$; 
therefore the flow in this region will be in equilibrium to all algebraic orders in $T_{a}^{-1}$. This is not the case if $\beta<1$, because then the temperature reaches its maximum value within the reaction zone, and the flow outside will be chemically frozen, to all algebraic orders in $T_{a}{ }^{-1}$; the fuel and oxidizer after crossing the reaction zone will coexist in frozen flow. If, in the first approximation, equilibrium flow exists on one side of the flame it is because, to this approximation, complete combustion of one of the reactants occurred in the reaction zone before the temperature decreased, toward the near-equilibrium side, enough to freeze the chemical reaction. However, leakage of this reactant through the flame will occur in all higher order approximations.

As mentioned before, the asymptotic solution for large $T_{a}$ of Eq. (6) is given in this regime by Eqs. (16) to (19) independently of chemical kinetics if $T_{p}$ is specified. To obtain higher order approximation to the flame structure and the relation between $T_{p}$ and the Damköhler number we shall proceed as in the previous regime. The detailed analysis that follows corresponds to the case $\alpha+$ $2 \beta<1$ in which no leakage of oxidizer through the flame occurs in the first approximation. The analysis is completely analogous, and the results may be directly written, for the case $\alpha+2 \beta>1$.

The problem we pose is to find the Damköhler number and the asymptotic solution of Eqs. (6) and (7), for low values of $\epsilon=T_{p}^{2} / T_{a}$, when the temperature in the frozen flow region, $\left(x_{\mathrm{p}}-x\right) / \epsilon \gg 1$, to the left of the reaction zone is given by Eq. (19) with $x_{p}$ and $T_{p}$ related by

$$
x_{p}=\left(T_{\infty}+\alpha-T_{p}\right) /(\alpha+\beta)
$$

For the temperature distribution in the near-equilibrium region, $\left(x-x_{p}\right) / \epsilon \gg 1$, to the right of the reaction zone, we use the expansion

$$
T=T_{\infty}-\beta+(\alpha+\beta)(1-x)+\epsilon P_{1}(1-x)+\epsilon^{2} P_{2}(1-x)+\cdots,
$$

in which we hypothesize that chemical equilibrium, with $y_{0}=0$, occurs in the first approximation. However, the chemical reaction will be frozen to all algebraic orders in $\epsilon$, because the temperature in this region will be lower than $T_{p}$ and the Damköhler number is not high enough to offset the effect of the Arrhenius exponent. Therefore leakage of the oxidizer through the flame occurs in the second approximation, proportional to

$$
-\left(d y_{0} / d x\right)_{x=1}=-\epsilon P_{1}-\epsilon^{2} P_{2}+\cdots
$$

For the analysis of the reaction zone we use the inner variable

and the expansion

$$
\eta=\alpha\left(x-x_{p}\right) / x_{p} \epsilon-p_{0} / m
$$

$$
T=T_{p}-\epsilon\left\{m \eta+p_{0}+y_{1}(\eta)\right\}-\epsilon^{2} y_{2}(\eta)+\cdots
$$

where

$$
m=x_{p}(1+\beta / \alpha)=\left(T_{\infty}+\alpha-T_{p}\right) / \alpha,
$$

$(m-1) / m$ is the ratio of the slopes of the temperature distribution to the left and right of the reaction zone; and $p_{0}$, which will be a function of $T_{p}$, which we 
anticipate to be of order unity, is the first term of an expansion

of

$$
p=p_{0}+p_{1} \epsilon+\cdots
$$

$$
p=\ln \left\{4 \pi \exp \left(z_{p}^{2}\right) y_{F p}\left(x_{p} T_{p}^{2} / \alpha T_{a}\right)^{2} D \exp \left(-T_{a} / T_{p}\right)\right\}
$$

The first approximation for the fuel concentration $y_{F p}$ appearing in Eq. (50) is

$$
y_{F p}=(1+\alpha) x_{p}-\alpha
$$

and $z_{p}$ is the value of $z$ corresponding to $x_{p}$.

If we introduce the expansions (47) and (49) into Eq. (6) previously written in terms of $\eta$, we obtain the equation, free from convective effects, for $y_{1}$,

$$
2 d^{2} y_{1} / d \eta^{2}=y_{1} \exp -\left(y_{1}+m \eta\right)
$$

Equation (52) is to be solved with the boundary conditions obtained from the matching conditions with the outer solutions given by Eq. (19) and (44). We therefore require,

$$
\begin{array}{lll}
\eta \rightarrow-\infty & : & d y_{1} / d \eta=-1 \\
\eta \rightarrow+\infty & : & d y_{1} / d \eta=0
\end{array}
$$

The choice of the stretching factor for the inner variable was made in order to obtain the normalized boundary condition (53). Similarly the choice of the translation $p_{0} / m$ was made so as to obtain the factor 2 in the left hand side of Eq. (52). This is the only value for which Eq. (52) has solutions satisfying the boundary conditions (53) and (54) in the limiting case $m=0$.

Figure 6 shows $y_{1}(\eta)$, for different values of $m$, as obtained from a numerical integration of Eq. (52). The results are also given for negative values of $m$, because in the analysis of the premixed flame regime for values of $\beta$ larger than one we encounter negative values of $m$. Notice that the exponential factor $\exp (-m \eta)$ in Eq. (52) represents the effect of the heat loss, or gain, from the reaction zone toward the near-equilibrium side of the flame.

Figure 7 shows the limiting values,

and

$$
n=\lim \left(y_{1}+\eta\right), \quad \text { for } \eta \rightarrow-\infty
$$

$$
y_{1 \infty}=\lim y_{1}, \quad \text { for } \eta \rightarrow \infty
$$

as a function of $m$.

For small values of $m, n=1.344$ and $y_{1 \infty}=0$. Both $y_{10}$ and $-n$ grow to infinity when $m$ approaches 0.5 , and there is no solution of Eq. (52), with the boundary conditions Eqs. (53) and (54), for $m>0.5$. The limiting value of $m$ correspond to equal rate of decrease of the temperature on both sides of the reaction zone. The temperature must, therefore, decrease at a slower rate toward the nearequilibrium side than toward the frozen flow side, so that matching is possible between the inner diffusive-reactive solution of Eq. (52) and the first approximation for the outer solutions.

When $m$ approaches 0.5 , as shown in Appendix $C, y_{100}$ and $-n$ grow toward infinity as $1 /(1-2 m)$ and $\left\{(1-2 m)^{-1}+2 \ln (1-2 m)\right\}$ respectively. For negative 


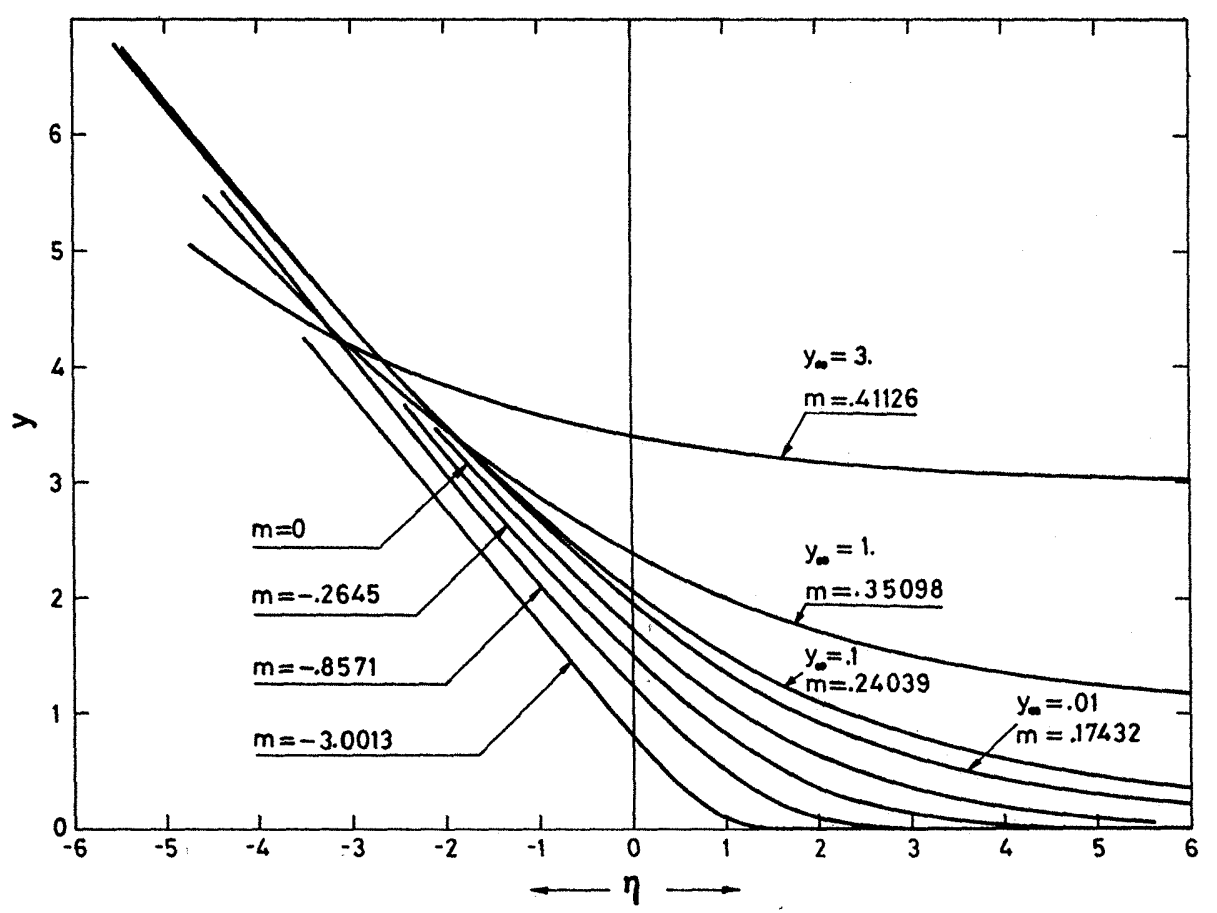

Fig. 6. Internal structure of the reaction zone for the premixed flame regime. The nondimensional reactant concentration, $y_{1}$, is plotted as a function of a nondimensional coordinate normal to the flame, $\eta$, for several values of the parameter $m$. The corresponding leakage of the reactant, $y_{1 \infty}$, has also been indicated in the figure.

values of $m, y_{1 \infty} \equiv 0$. For large negative values of $m, n$ behaves as $\left\{-\ln \left(2 m^{2}\right)+\right.$ $\left.2 \gamma_{E}\right\} / m$, where $\gamma_{E}$ is the Euler constant $\gamma_{E}=0.577$; and the solution $y_{1}(\eta)$ takes the form $y_{1}=-(2 / m) K_{0}(t)$, where $K_{0}(t)$ is the modified Bessel function of order zero and $t=-(\sqrt{2} / m) \exp (-m \eta / 2)$.

The matching conditions yield the anticipated boundary conditions (53) and (54), and in addition provide the relations

and

$$
p_{0}=-n m
$$

$$
P_{1}\left(1-x_{p}\right)=-y_{100}
$$

Equation (57) is the desired relation between the first approximation for the Damköhler number $D_{\mathrm{p}}$ and the flame temperature. Equation (58) gives the amount of oxidizer leaking through the flame, which therefore is of order $\epsilon$.

We write Eq. (57) explicitly as

$$
D_{p} y_{F p} \exp \left(-T_{a} / T_{p}\right)=(1 / 4 \pi)\left(\alpha T_{a} / x_{p} T_{p}^{2}\right)^{2} \exp \left(-z_{p}{ }^{2}-n m\right)
$$

Here $n m$ is a function of $m$, and therefore of $T_{p}$, which has also been represented in Fig. 7. In the case $\alpha+2 \beta<1$ that we have considered $m$ decreases from $1 / 2$ to 


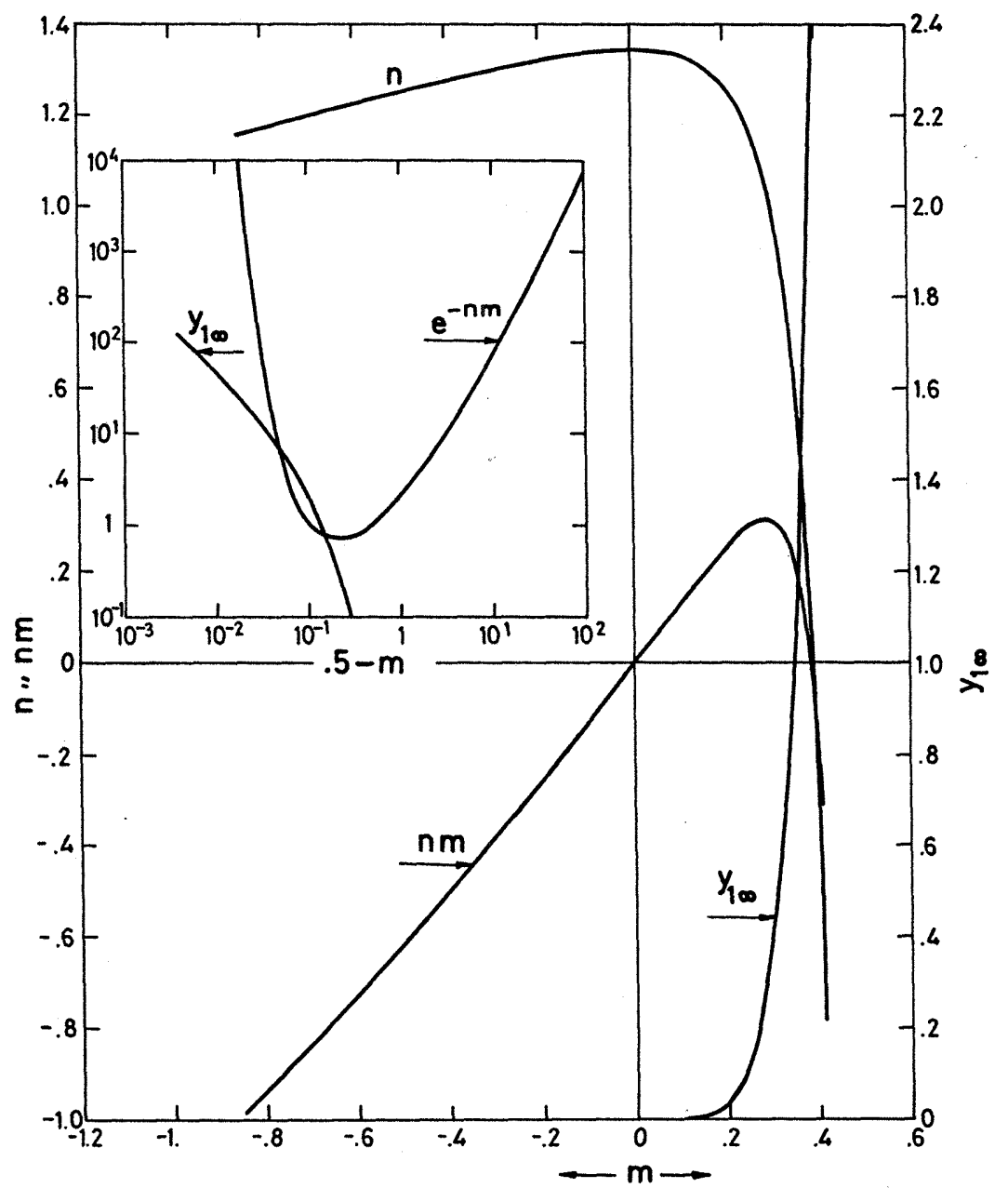

Fig. 7. The parameters $y_{1 \infty}$ and $n$, defining the asymptotes of the relation $y_{1}(\eta)$, are here shown as functions of $m$. Notice that $y_{1 \infty}=0$ for $m<0$.

$(\alpha+\beta) /(\alpha+1)$, when $T_{p}$ increases from its lower bound $T_{\infty}+\alpha / 2$ to its upper bound $T_{e}$.

We shall now present the results corresponding to the case where $\alpha+2 \beta>1$, for which nearly complete consumption of the fuel takes place in the thin reaction zone.

In the first approximation, the flame temperature $T_{p}$, the flame location $x_{p}$, and the oxidizer concentration $y_{0 p}$ are related by

$$
\begin{aligned}
x_{p} & =\left(T_{p}-T_{\infty}\right) /(1-\beta) \\
y_{0 p} & =\alpha-(\alpha+1) x_{p}
\end{aligned}
$$

For the analysis of the reaction zone we introduce the inner variable

$$
\eta=-(1-\beta)\left(x-x_{p}\right) / m \epsilon-p_{0} / m
$$


where $m$ is now, given by

$$
m=1-\left(T_{p}-T_{\infty}+\beta\right)
$$

and to define $p$, instead of Eq. (50), we use

$$
p=\ln \left\{4 \pi \exp \left(z_{p}^{2}\right)\left(m T_{p}^{2} / T_{a}\right)^{2}(1-\beta)^{-2} D_{y_{p}} \exp \left(-T_{a} / T_{p}\right)\right\}
$$

We use Eq. (47) as the inner expansion, and Eq. (17) for the temperature distribution to the right of the reaction zone. For $x<x_{p}$ we use the expansion

$$
T=T_{\infty}+(1-\beta) x+\epsilon P_{1} x+\epsilon^{2} P_{2} x+\cdots
$$

We then obtain Eqs. (52) to (54) for $y_{1}(\eta)$, and from the matching conditions Eq. (57) and

$$
P_{1} x_{p}=-y_{1 \infty} \text {. }
$$

Then, the following relation is finally obtained between the flame temperature, $T_{p}$, and the first approximation, $D_{p}$, for the Damköhler number

$$
D_{p} y_{0 p} \exp \left(-T_{a} / T_{p}\right)=(1 / 4 \pi)\left\{m T_{p}^{2} / T_{a}(1-\beta)\right\}^{-2} \exp \left(-z_{p}^{2}-n m\right)
$$

When $\beta<1 / 2$, the flame temperature is, in this regime, bounded by $T_{\infty}-\beta+$ $1 / 2<T_{p}<T_{e}$, and therefore, $1 / 2>m>(1-\beta) /(1+\alpha)$. When $\beta>1 / 2, T_{\infty}$ and $T_{e}$ are the limiting values of $T_{p}$, so that $1-\beta$ and $(1-\beta) /(1+\alpha)$ are the corresponding limits of $m$; we thus find $m$ to be negative only if $\beta>1$, and zero of $\beta=1$.

In the particular case $\beta=1$, the flame temperature $T_{p}$ is, in this regime, equal to $T_{\infty}$, so that $m=0$; then we should use $x_{p}$ as the independent variable. The factor $(1-\beta) / m$ appearing in Eqs. (62), (64) and (67) must be substituted by $\left(1-x_{p}\right)^{-1}$; and in this case $p_{0} / m=-n=-1.344$.

It is clear that this premixed flame analysis will not be valid for $m$ close to its upper limit $1 / 2$, because then both $p_{0} / m=-n$ and $y_{1}$ become infinite; leakage of the lean reactant through the flame increases, so that the equilibrium approximation on one side of the flame cannot be used as the first order approximation. The partial burning analysis of the previous reaction should be used for $m>1 / 2$.

When $T_{p}$ becomes close to $T_{e}$ the concentrations of both reactants in the reaction zone are small and have relative variations of order unity; then we must use the analysis of the near-equilibrium, diffusion flame regime that follows. The extinction conditions occur very often under the premixed flame regime.

When $\beta>1 / 2, T_{\infty}$ is a bounding value of $T_{p}$, and the premixed flame analysis should be replaced by the analysis of the nearly frozen regime if $\left(T_{p}-T_{\infty}\right)$ becomes small. The ignition condition may also occur under the premixed flame regime if $(1-\beta)$ is small.

\section{Near equilibrium, diffusion flame, regime}

Finally, a diffusion controlled regime exists for which the flow is everywhere near equilibrium, so that in a first approximation the flame position and temperature distribution are determined by Eqs. (20) to (23) independently of chemical kinetics. This corresponds to the Burke-Schumann[18] and classical diffusion flame analysis [19]. 
We shall evaluate below, for large activation energies, the non equilibrium effects in the range of Damköhler numbers for which the temperature deviations from equilibrium are of order $\epsilon=T_{e}^{2} / T_{a} \ll 1$. The nonlinear effects associated with the Arrhenius exponent are responsible for the existence if $\beta<1$ of extinction conditions, which occur in this regime if $(1-\beta)$ is not very small. By extinction conditions we mean that multiple solutions of Eq. (6) exist for Damköhler numbers above an extinction value, and only a nearly frozen solution exists for smaller values of $D$. Because the Damköhler number is a single valued function of the deviations of the temperature from equilibrium, and not necessarily vice versa, we shall pose our problem as to find $D$ and the asymptotic solution of Eqs. (6) and (7), for a deviation, of order $\epsilon$, of the temperature from its equilibrium value at a given point, $x=x_{e}$ for example.

We introduce the outer expansion

$$
\begin{aligned}
& T=T_{\infty}+(1-\beta) x-\epsilon A_{1} x-\epsilon^{2} A_{2} x+\cdots \\
& T=T_{\infty}+\alpha-(\alpha+\beta) x-\epsilon B_{1}(1-x)-\epsilon^{2} B_{2}(1-x)+\cdots
\end{aligned}
$$

for large negative and positive values of $\left(x-x_{e}\right) / \epsilon$, respectively. When writing these expansions we assume, in the first approximation, chemical equilibrium with $y_{F}=0$ or $y_{0}=0$. We, in addition, anticipate that in the outer regions we have, to all algebraic orders in $\epsilon$, frozen flow, or equilibrium flow if $A_{i}=B_{i}=0$.

For the temperature distribution in the reaction zone we introduce the expansion

$$
T=T_{e}-\delta_{0}^{-1 / 3}\left(\epsilon \beta_{1}+\epsilon \gamma \xi+\epsilon^{2} \beta_{2}+\cdots\right),
$$

where $\beta_{1}, \beta_{2}, \cdots$, are functions of the inner variable

$$
\xi=\delta_{0}^{1 / 3}(\alpha+1)\left(x-x_{e}\right) / 2 \epsilon .
$$

In Eq. (70) the parameter

$$
\gamma=1-2(1-\beta) /(1+\alpha)=2(\alpha+\beta) /(1+\alpha)-1
$$

is such that $(1-\gamma)$ is two times the ratio between the heat lost from the flame toward the region of negative $\left(x-x_{e}\right)$ and the total chemical heat release at the flame. When $\gamma=0$ the heat losses from the flame toward both sides are equal: when $\gamma>0$ the temperature decreases faster toward the region $x>x_{e}$ than toward the region $x<x_{e}$. For $\gamma>1$ the flame receives heat by heat conduction from the region $x-x_{e}<0$. The parameter $\delta_{0}$, which we anticipate to be of order unity, is the first term of an expansion,

$$
\delta=\delta_{0}+\epsilon \delta_{1}+\cdots,
$$

of the reduced Damköhler number

$$
\delta=8 \pi \exp \left(z_{e}^{2}\right)\left(T_{e}^{2} / T_{a}\right)^{3}(\alpha+1)^{-2} D \exp \left(-T_{a} / T_{e}\right)
$$

The length scale for the inner variable and the definition of the reduced Damköhler number $\delta$ were chosen so as to obtain the following equation for $\beta_{1}$,

$$
d^{2} \beta_{1} / d \xi^{2}=\left(\beta_{1}-\xi\right)\left(\beta_{1}+\xi\right) \exp \left\{-\delta_{0}^{-1 / 3}\left(\beta_{1}+\gamma \xi\right)\right\},
$$


where no convective effects appear, to be solved with the conditions,

$$
\begin{array}{lll}
d \beta_{1} / d \xi=1 & \text { for } & \xi \rightarrow \infty \\
d \beta_{1} / d \xi=-1 & \text { for } & \xi \rightarrow-\infty
\end{array}
$$

obtained from the matching conditions between the inner and outer expansions. The matching conditions do also provide the relations

and

$$
A_{1} x_{e} \delta_{0}{ }^{1 / 3}=\lim \left(\beta_{1}+\xi\right), \quad \text { for } \quad \xi \rightarrow-\infty
$$

$$
B_{1}\left(1-x_{e}\right) \delta_{0}{ }^{1 / 3}=\lim \left(\beta_{1}-\xi\right), \quad \text { for } \quad \xi \rightarrow \infty
$$

which enable us to calculate the distribution of reactants leaking through the reaction zone.

Equations (75) to (77) describe in the first approximation the non equilibrium effects, as dependent only on the reduced Damköhler number and $\gamma$. The solutions for positive and negative $\gamma$ are trivially related, so we shall consider below $\gamma \geq 0$.

Higher order approximations could be determined by insuring, for example, that the temperature obtained from the two term expansion at $\xi=0$ is not modified by higher order effects. We shall not calculate them here.

The retention in Eq. (75) of the exponential Arrhenius factor is essential for evaluating the non equilibrium effects in the near-extinction conditions. Eq. (75) with the boundary conditions (76) and (77) has two solutions, if $|\gamma|<1$, for $\delta_{0}$ above an extinction value $\delta_{0 E}(\gamma)$, and no solutions for $\delta_{0}<\delta_{0 E}$. Zeldovich [13] showed, by an order of magnitude analysis, the importance of the Arrhenius exponent in determining the extinction conditions, and indicated how the exponent could be linearized around the adiabatic flame temperature if the activation energy was sufficiently large. Librovich [20], independently, obtained and solved Eq. (75) in the particular case $\gamma=0$.

For large values of $\delta_{0}$, there is a solution of Eq. (75) that tends toward the solution of the equation

$$
d^{2} \beta_{1} / d \xi^{2}=\left(\beta_{1}{ }^{2}-\xi^{2}\right)
$$

with Eqs. (76) and (77) as boundary conditions. Eq. (80) was obtained in Refs. [14,21 and 22] as describing the structure of the reaction zone in diffusion flames in the limit of large Damköhler numbers. The decay to zero in this solution of $\left(\beta_{1}-\xi\right)$ and $\left(\beta_{1}+\xi\right)$, which are proportional to the oxidizer and fuel concentration, for large values of $|\xi|$, is exponential; thus to all algebraic orders there is no leakage of reactants through the flame for sufficiently large $\delta_{0}$.

A regular expansion of $\beta_{1}$ in $\delta_{0}^{-1 / 3}$ is not convergent for $\delta_{0}$ of order unity so that a numerical integration of Eqs. (75) was carried out. The results as summarized in Figs. 8 to 12 indicate that:

(a) If $\gamma<1$, there are no solutions of Eq. (75) for $\delta_{0}$ lower than $\delta_{0 E}(\gamma)$, and two solutions for $\delta_{0}>\delta_{0 \mathrm{EE}}$. Thus, we find for $\gamma<1$ a minimum Damköhler number for the existence of a near-equilibrium solution, and this may be used as an extinction criterium. When $\gamma$ approaches 1 from below $\delta_{E}$, goes to zero. When $\gamma>1$ the solution is unique. 
NEAR-EQUIL. DIFF. FLAME STRUCTURE

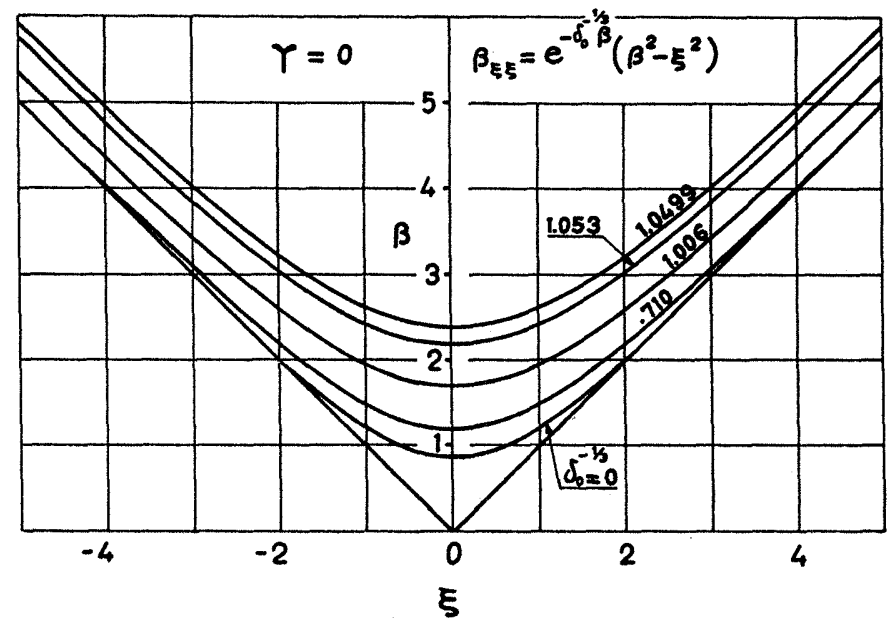

Fig. 8. The internal structure of the reaction zone in the near-equilibrium, diffusion controlled, regime for the case $\gamma=0$. No solution exists for $\delta_{0}^{-1 / 3}>1.053$, and two solutions exist for $\delta_{0}^{-1 / 3}<1.053$.

\section{NEAR-EQUILIBRIUM DIFFUSION FLAME STRUCTURE}

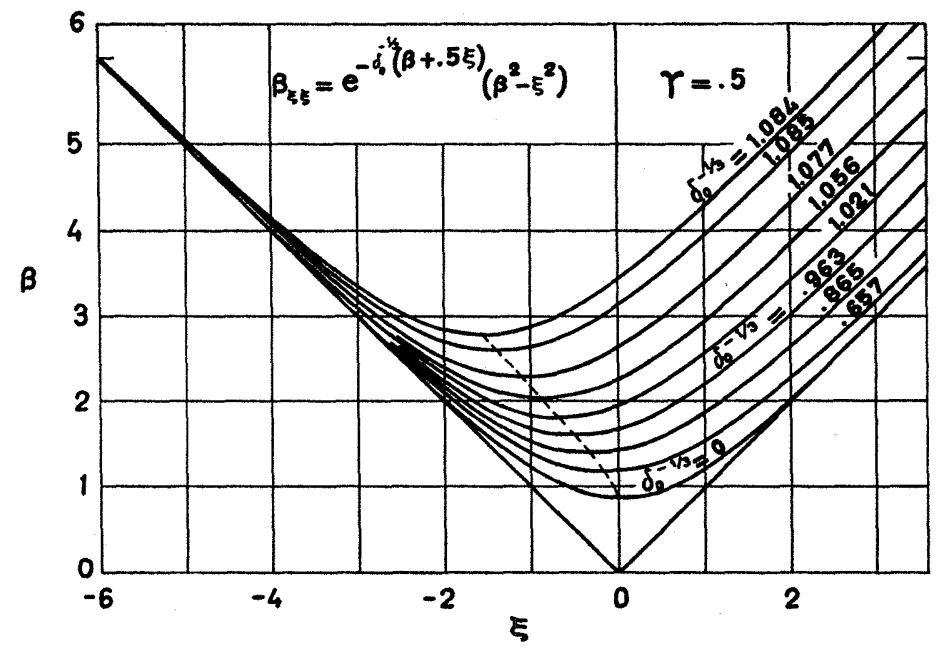

Fig. 9. Same as figure 8 , for the case $\gamma=0.5$. No solutions exist for $\delta_{0}{ }^{-1 / 3}>1.077$. The premixed flame character of the reaction zone for the Damköhler numbers close to the extinction one is already apparent for this value of $\gamma$.

(b) There is leakage of both reactants through the flame as evidenced from the fact that $\left(\beta_{1}-\xi\right)_{\infty}$ and $\left(\beta_{1}+\xi\right)_{-\infty}$ are, functions of $\gamma$ and $\delta$, different from zero. For $\gamma \geq 1,\left(\beta_{1}+\xi\right)_{-\infty}$ is identically zero. For $\gamma=0,\left(\beta_{1}-\xi\right)_{\infty}$ and $\left(\beta_{1}+\xi\right)_{\infty}$ are, of course, equal. When $\gamma$ increase from zero to one, for fixed $\delta_{0},\left(\beta_{1}+\xi\right)_{-\infty}$ goes to zero and $\left(\beta_{1}-\xi\right)_{\infty}$ goes to infinity. 
NEAR-EQUIL. DIFF. FLAME STRUCTURE

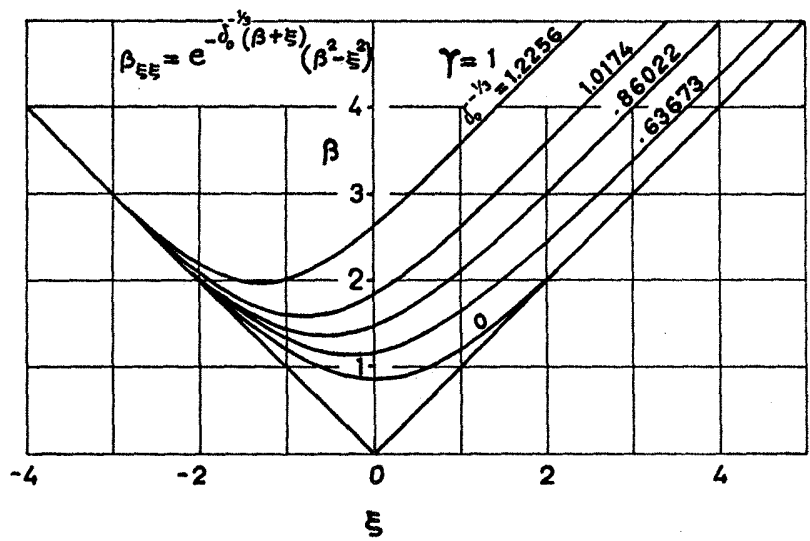

Fig. 10. Diffusion flame structure for the case $\gamma=1$. No Damköhler number of extinction exists in this case, as it occurs for $|\gamma|>1$. There is not leakage of one reactant through one side of the flame, $(\beta+\xi)_{-\infty}=0$. For the lower values of $\delta_{0}$, the reaction zone structure is of the premixed flame type.

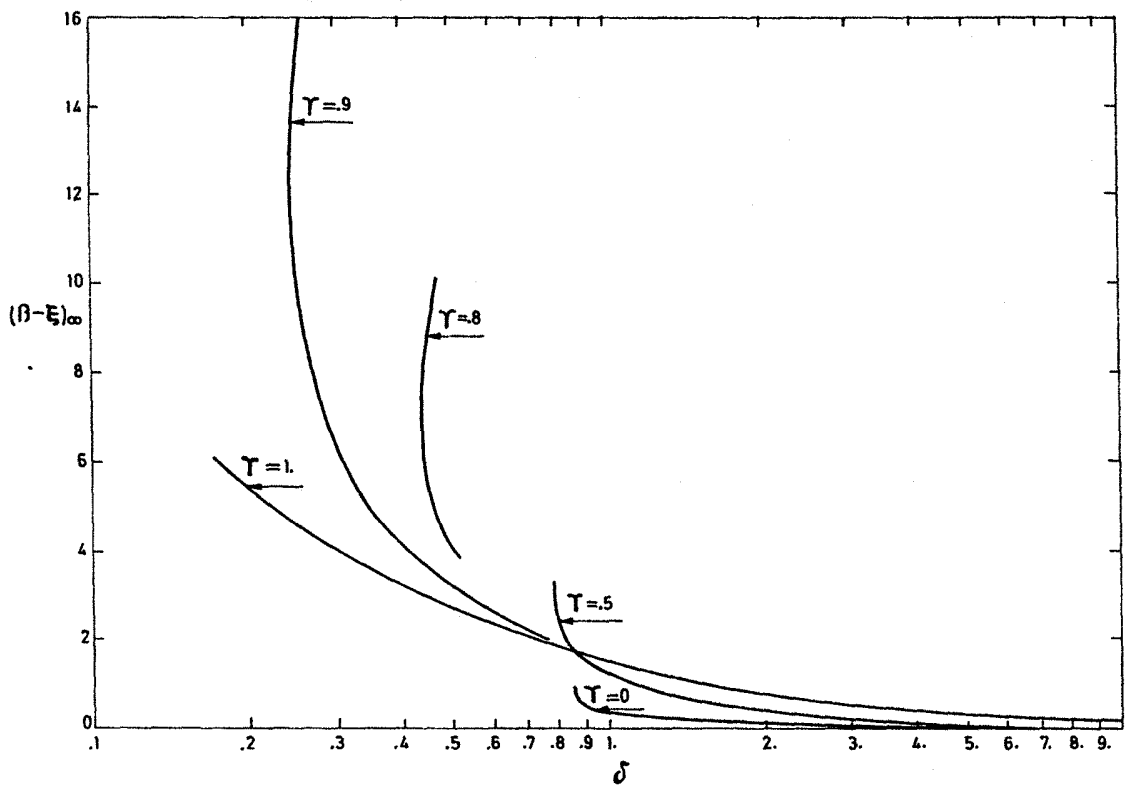

Fig. 11. The nondimensional reactant leakage $(\beta-\xi)_{\infty}$ is plotted in terms of the reduced Damköhler number $\delta_{0}$ for several values of $\gamma$, in the near-equilibrium regime. The solution is found to be double-valued for $\delta_{0}>\delta_{E}(\gamma)$ if $\gamma<1$.

(c) The leakage $\left(\beta_{1}-\xi\right)_{\infty}$ is larger for the unstable branch than for the nearequilibrium stable branch. For large $\delta_{0}$ the solution corresponding to the unstable branch shows a thin reaction zone, of the premixed flame type, at large negative values of $\xi$. 
(d) For $\gamma$ close to 1 the extinction regime occurs at small values of $\delta_{0}$, with a thin reaction zone centered at a large negative $\xi=-\left(\beta_{1}-\xi\right)_{\infty} / 2$. In the reaction zone $\left(\beta_{1}-\xi\right)$ stays practically constant, and $\beta_{1}+\xi$ decays to zero for large negative $\xi$. That is, the reaction zone is of the premixed flame type and the analysis of the previous section may be used, resulting in

$$
\delta_{0 E}=e(1-\gamma)
$$

An expansion for small values of $(1-\gamma)$ of the solution of Eq. (75) may be shown [23] to yield (81) in a first approximation, and the first two terms of

$$
\delta_{0 E}=e\left\{(1-\gamma)-(1-\gamma)^{2}+0.26(1-\gamma)^{3}+0.055(1-\gamma)^{4}\right\}
$$

in a second approximation. Eq. (82) is seen from Fig. 12 to be a very good approximate representation of the numerical result $\delta_{0 E}(\gamma)$, for all values of $\gamma>$ 0.5 , and its error is less than one per cent for lower $\gamma$. For negative values of $\gamma$, calculate $\delta_{0 E}$ using Eq. (82) with $\gamma$ replaced by $|\gamma|$.

The Damköhler number for extinction is obtained by replacing $\delta$ by $\delta_{0 E}$ in Eq. (74).

However, the deviations of the temperature $T$ from equilibrium, obtained in

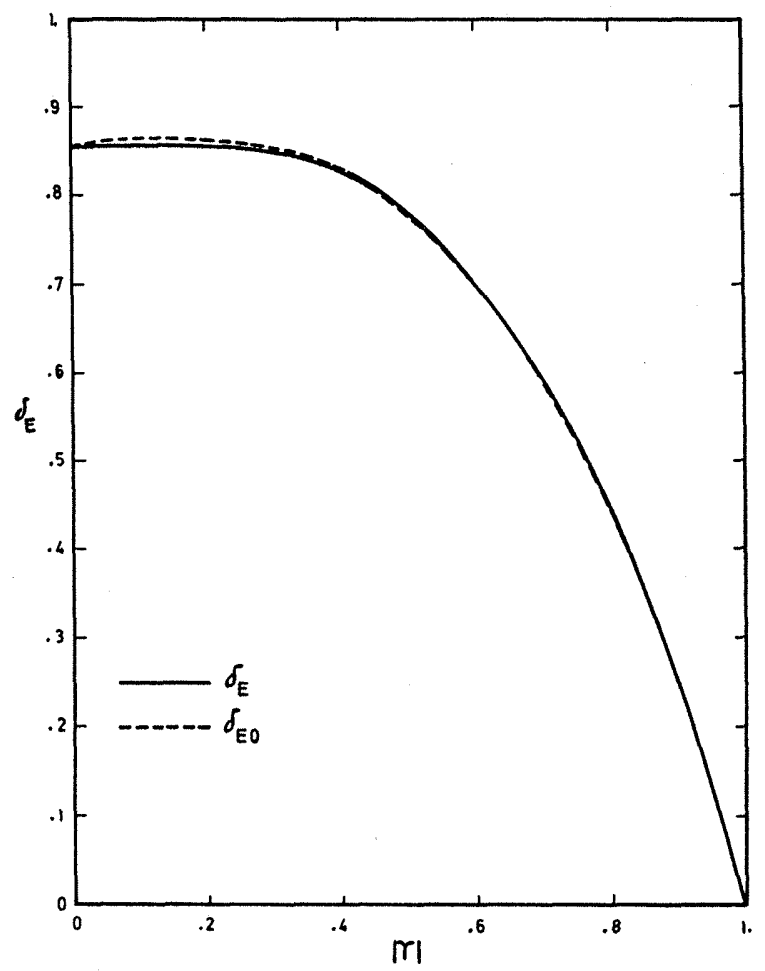

Fig. 12. The first approximation for the Damköhler number of extinction $\delta_{E}$ is shown as a function of $|\gamma|$, as obtained from the numerical solutions (solid line) and as given by Eq. (82) (dashed line). 
this near-equilibrium analysis, for small values of $(1-\gamma)$ are of order $T_{e}^{2} / T_{a}(1-$ $\gamma)$. Hence, to predict the extinction condition for small values of $(1-\gamma)$, the analysis of the previous section should be used, unless the activation energy becomes sufficiently large.

\section{Discussion and generalization}

The analysis of the previous sections covers all the regimes that can be found when analysing diffusion flames, unless they are of the unsteady or evolution type [25], or the chemical reaction is multi-step and does not admit a simplified description by a single overall irreversible reaction.

The non-dimensional activation energy, or more precisely $T_{a} / T_{r}^{2}$ (where $T_{r}$ is the characteristic temperature of the reaction zone), has been used as the large parameter in the asymptotic analysis. It is interesting to observe that the parameter $T_{a} / T_{r}^{2}$ is the product of the nondimensional activation energy and the nondimensional heat release, or third Damköhler number, both based on the characteristic thermal energy at the temperature of the reaction zone.

The first Damköhler number, or nondimensional frequency factor, is allowed to grow appropriately with $T_{a} / T_{r}^{2}$ so as to obtain nonzero heat release rates in the limit of infinite $T_{a} / T_{r}^{2}$. In this limit the chemical reaction takes place only at a well defined temperature $T_{r}$, which is then used in the analysis of the partial burning and premixed flame regimes as the independent variable to calculate the corresponding Damköhler number $D$.

As a result of the analysis the temperature and concentration distributions have been obtained in terms of the five parameters $D, T_{a}, T_{\infty}, \beta$ and $\alpha$ for the four possible regimes. A detailed parametric discussion of the results will not be undertaken here. We shall give only a brief discussion of some of the results.

In particular Eqs. (41) and (59) provide the relations, for the partial burning regimes and for the premixed flame regimes, between the asymptotic flame temperature and the Damköhler number. $\dagger$ Both curves, $T_{b}(D)$ and $T_{p}(D)$, have branches that exhibit an increasing temperature with decreasing Damköhler numbers. A stability analysis would very likely show these branches to be unstable.

Both curves give an infinite Damköhler number at a common transition temperature. The analyses given for both regimes fail at temperatures close to the transition temperature. An appropriate asymptotic analysis of a transition regime would provide a smooth transition between the valid results of the partial burning and premixed flame regimes. The analysis is not included because this transition regime will always be unstable (as is also the case with the partial burning regime).

Figure 13 shows the relation $T_{p}(D)$ obtained from the asymptotic analysis of the premixed flame regime, for some values of the parameters and indicates how the Damköhler number changes with changes in $\beta$ and $T_{a}$. The lower branch of these curves is unstable. An extinction Damköhler number can be obtained from curves such as these as a function of $\beta, \alpha, T_{\infty}$ and $T_{a}$.

†This relation can be simplified by using the following approximate relation for $E=2 \pi \exp \left(z^{2}\right)$ as a function of $x: E^{-1}=x^{2} \ln \left\{(1+c x) / 2 \pi x^{2}\right\}$ for $0<x \leq 0.5$ and the symmetric relation for $0.5 \leq x<1$, where $c=3.938$. 


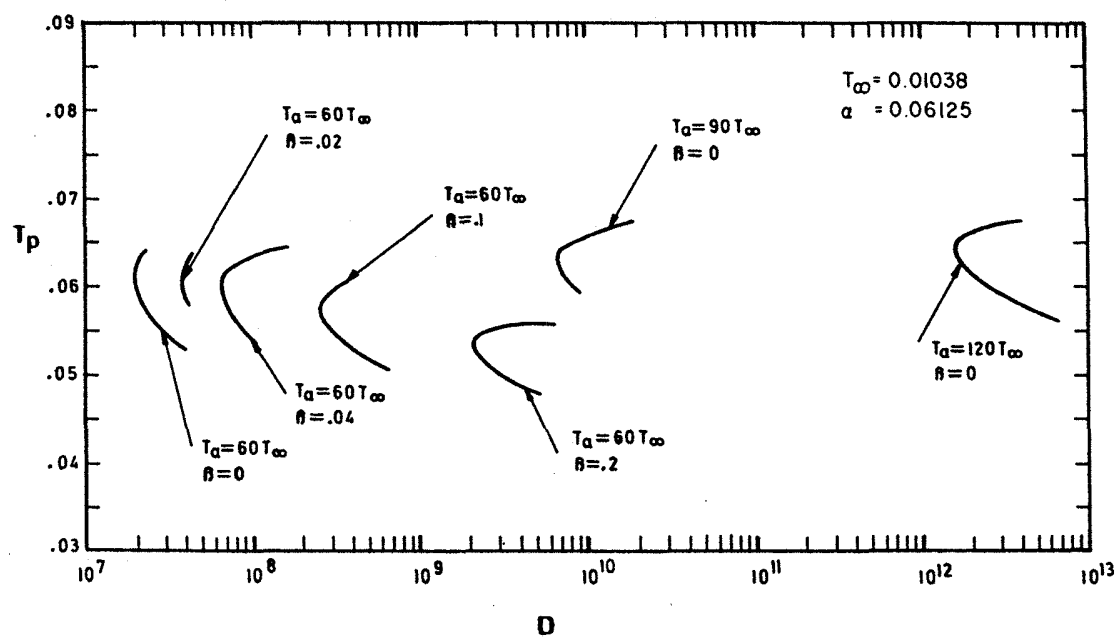

Fig. 13. The first approximation $T_{p}$ of the flame temperature in the pre-mixed flame regime, for various activation energies and differences in initial temperature of the reactants, as functions of the Damköhler number, for Damköhler number close to the extinction one.

The premixed flame analysis covers a wide range of stable non-equilibrium regimes in non-premixed systems. The relations (59) or (67) may be used to predict the flame characteristics whenever $|\gamma|$ is larger than or moderately close to 1 . In these expressions $n m$ is given as a function of $m$ in Fig. 7, and $z_{p}$ is related to $x$ by Eq. (5). These relationships may be simplified by use of the correlation given at the end of Appendix $C$ and the approximate expression for exp $\left(z^{2}\right) \dagger$ With very little loss of accuracy we may write $n=1.344$ in Eq. (59) or (67) to deduce the value of $n$ as a function of $m$. For the extinction conditions to occur $|\gamma|$ must be lower than one. If in this case $|\gamma|$ is close to one, the extinction conditions occur with a premixed flame regime, otherwise they occur under the near-equilibrium regime.

Figure 14 , shows $-y_{0}^{\prime}(0) / \alpha$, which turns out to be proportional to the mass consumption of the oxidizer, as a function of the Damköhler number, calculated employing the reaction-rate function used by Jain and Mukunda [6]. Results for ignition, partial-burning and premixed-flame regimes appear, as well as a comparison of extinction Damköhler numbers with their numerical results. Agreement is seen to be excellent. In the equilibrium limit, $-y_{0}^{\prime}(0) / \alpha$ approaches $1 / \alpha$, which is 16.3 in the present case. Therefore Fig. 14 demonstrates that typically under extinction conditions there is a large reduction in the consumption of the oxidant at the flame due to nonequilibrium effects. We should expect larger changes in oxidant consumption and in flame position than in flame temperature.

From Figs. 8 to 12 it may be seen that leakage of one of the reactants through the flame is possible even in the strongly burning stable branch of the nearequilibrium regime, for Damköhler numbers above extinction. Kassoy and 


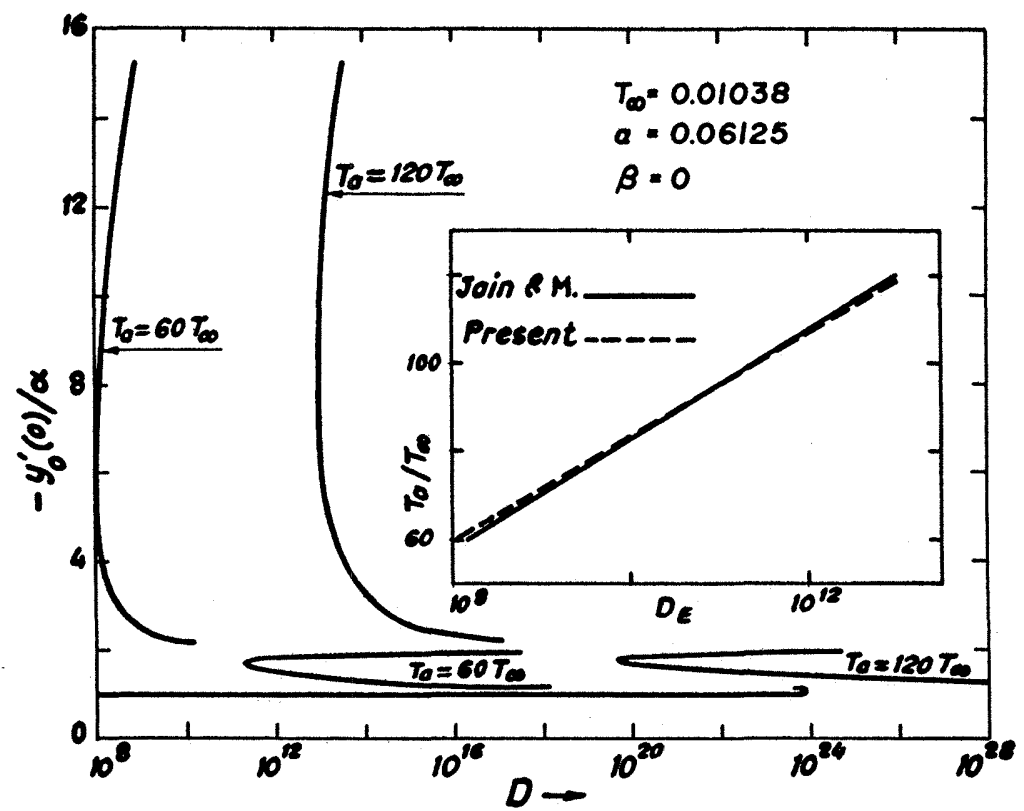

Fig. 14. The oxidizer consumption rate at the flame $\left(-y^{\prime}(0) / \alpha\right)$ is plotted in terms of the Damköhler number as obtained from the asymptotic analysis of the premixed flame regime, the partial burning regime, and the nearly frozen regime. The asymptotic Damköhler number of extinction is compared with the one obtained numerically by Jain and Mukunda.

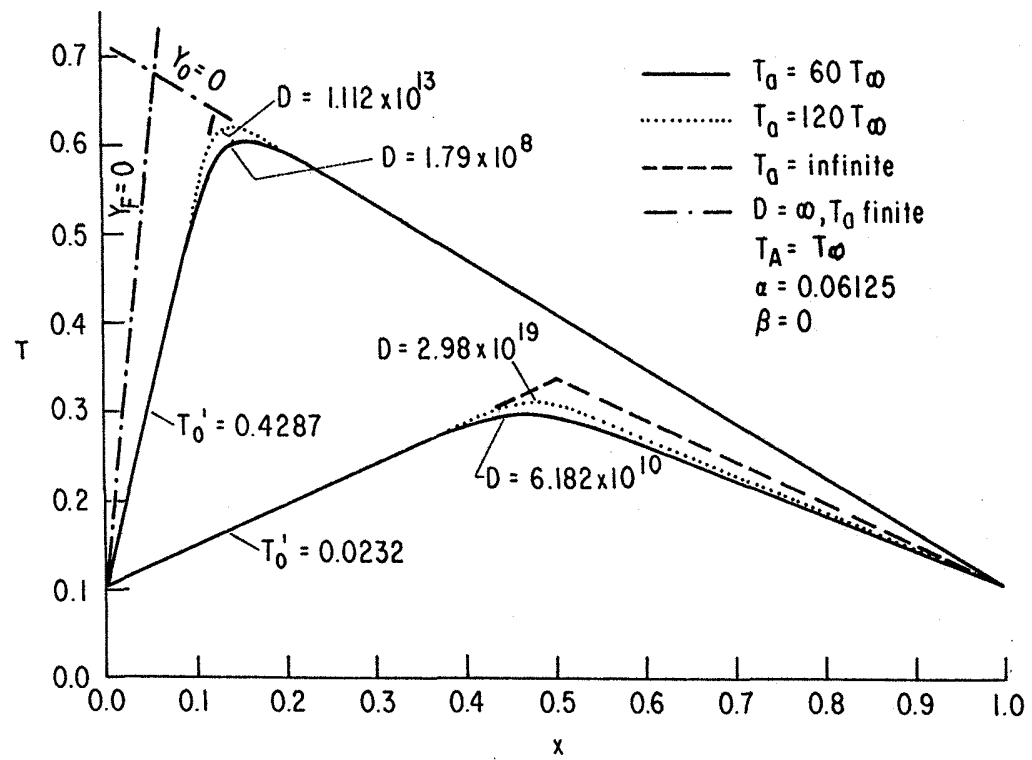

Fig. 15. Temperature distribution corresponding to two values of the heat flux from the oxygen-side, leading to the premixed flame regime and the partial burning regime. 
Williams [11] obtained a similar leakage of reactants through a near-equilibrium diffusion flame, associated with orders of the reaction with respect to the reactants different from unity.

Figure 15 shows temperature profiles obtained by numerical integration for two oxygen-side fluxes, one corresponding to the premixed-flame regime and the other to the partial-burning regimes. Curves are given for three nondimensional activation energies, showing how the asymptotic structure described in Section 2 is approached for very large activation energies. Again, rates of Jain and Mukunda [6] have been used. The equilibrium solution also is included, so that the correspondence with Fig. 3 becomes evident. The curves demonstrate thickening and some translation of the reaction zone.

The character of the results demonstrates clearly that the analysis can be extended to other fluid dynamical configurations and to include more realistic descriptions of the transport coefficients. The structures of the outer zones will change, but the key aspect of the analysis, namely the reaction-zone calculations and matching techniques developed herein, will be identical.

Acknowledgment-This research was partially sponsored by the Air Force Office of Scientific Research through its European Office, under contract No. AFOSR 72-2254. Most of the numerical calculations that play an important role in this paper were carried out by Mr. V. Torroglosa. A number of the ideas and concepts introduced in this paper became ordered and clear during many enjoyable hours of discussion with Professor Forman Williams of the University of California S.D.; I am also in debt to him for his encouragement in publishing this paper.

\section{Appendix A}

We analyze here the ignition regime for the case where $\beta$ is of order unity, or simply $\beta \gg T_{\infty}{ }^{2} / T_{a}=\epsilon$.

It is clear from Eq. (6), written here in terms of the variable

$$
\theta=T-T_{\infty}+\beta x \text {, }
$$

as

$$
d^{2} \theta / d x^{2}=-2 \pi \exp \left(z^{2}\right) y_{0} y_{F} D \exp \left(-T_{a} / T_{\infty}\right) \exp \left\{T_{a}(\theta-\beta x) / T_{\infty} T\right\},
$$

that the chemical reaction will be frozen except for small values of $x$, such as to make $\beta x$ of order $T_{\infty}{ }^{2} / T_{a}$. Otherwise the Arrhenius exponential factor will become extremely small. Notice that in the ignition regime $\theta$ is uniformly small, of order $\epsilon$.

For the analysis of the reaction region we introduce the inner variable

$$
\chi=\beta x / \epsilon
$$

and expand $T$ as

$$
T=T_{\infty}+\epsilon\left(\theta_{1}-\chi\right)+0\{\epsilon /(\ln \epsilon)\} .
$$

We shall take into account that for small $x$, or $\chi$ of order unity,

$$
2 \pi x^{2} z^{2} \exp \left(z^{2}\right) \doteq\left(1-2 / z^{2}+\cdots\right)
$$

and

$$
2 \pi \exp \left(z^{2}\right) \doteq \beta^{2}(\epsilon \chi)^{-2} z^{-2} \doteq \beta^{2}(\epsilon \chi)^{-2} z_{\epsilon}^{-2},
$$


where $z_{e}^{2}$ is a function of $\epsilon$ and $\beta$ satisfying the relation

$$
z_{\epsilon}^{2}=-\ln \left(2 \pi \epsilon^{2} \beta^{-2} z_{\epsilon}^{2}\right),
$$

so that for small $\epsilon, z_{\epsilon}^{2}$ is a large number, $z_{\varepsilon}^{2} \rightarrow-\ln \left(\epsilon^{2} \beta^{-2} 2 \pi\right)$.

By substituting Eqs. (A3) to (A6) into (A2) we obtain the following equation for $\theta_{1}$, if we neglect terms of order $z_{\epsilon}^{-2}$,

$$
\chi^{2} d^{2} \theta_{1} / d \chi^{2}=-\Delta\left(\chi-\beta \theta_{1}\right) \exp \left(\theta_{1}-\chi\right)
$$

where $\Delta$ is a reduced Damköhler number

$$
\Delta=\beta^{-1} z_{\epsilon}^{-2} \alpha D \exp \left(-T_{a} / T_{\infty}\right)
$$

Equation (A7) is to be solved with the boundary conditions

$$
\theta_{1}(0)=0, \quad \theta_{1 x}(\infty)=0
$$

The last condition is obtained when matching the inner expansion (A4) with an outer expansion,

$$
T=T_{\infty}-\beta x+\epsilon I_{1}(1-x)+\cdots,
$$

for the frozen flow region. The matching conditions provide, in addition, the relation $I_{1}=\theta_{1}(\infty)$.

To obtain higher order terms, a good procedure is to fix the expansion (A10), and determine $D$ in terms of $I_{1}$. We should then substitute for the $\Delta$ appearing in Eq. (A7), the first term of an expansion in $\epsilon$ of the $\Delta$ defined by Eq. (A8).

Equation (A7), with the boundary conditions (A9), has been integrated numerically [25] for several values of $\beta$. We obtained $\theta_{1}$ as a function of $\chi$ for some typical values of $\beta$ and different values of $\Delta$. From the numerical results we obtained $\theta_{1}(\infty)$ as a function of $\Delta$ and $\beta$, shown in Fig. 16. It turns out that if $\beta<1$

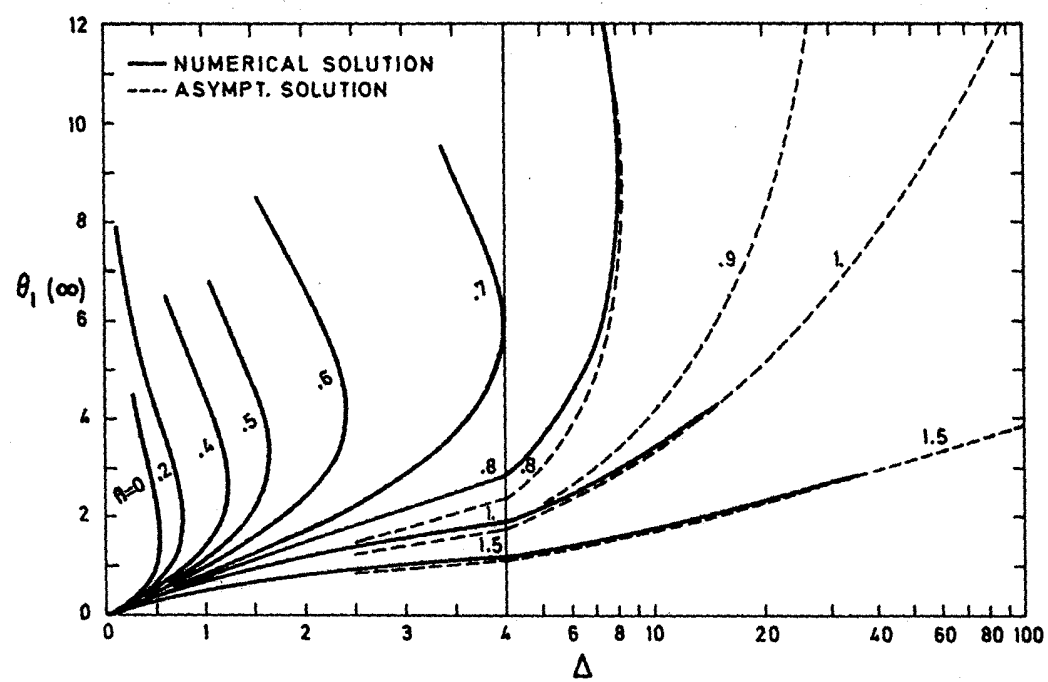

Fig. 16. The nondimensional maximum temperature increment associated with the chemical reaction in the nearly frozen, ignition, regime as a function of the Damköhler number $\Delta$ for several values of $\beta$. 
two solutions exist for each $\Delta$ lower than an ignition $\Delta_{I}(\beta)$ and no solution for $\Delta>\Delta_{l}$. We see reproduced in Fig. 16 the lower bend of the typical $S$ curves, relating maximum temperature and Damköhler number. For $\beta \geq 1, \theta_{1}(\infty)$ is uniquely related to $\Delta$.

An asymptotic analysis of Eq. (A7) was carried out by Liñán and Crespo[25] for large values of $\theta_{1}(\infty)$, using the ideas of Section 5. For large $\theta_{1}(\infty)$ a thin reaction zone located around $\chi_{r}=\beta \theta_{1}(\infty)$ separates a region of near-equilibrium, for lower $\xi$, from a region of frozen flow for $\xi-\xi_{r}>0$. This analysis can be used to calculate the Damköhler number of ignition for small values of $(1-\beta)$, because in first approximation $\theta_{1}(\infty)=2 /(1-\beta)$ at the ignition conditions. The first two terms of the expansion of $\Delta_{\mathrm{I}}$ in terms of $(1-\beta)$ yield the following expression for the Damköhler number of ignition

$$
D_{\mathrm{I}} \alpha e^{-T_{\alpha} / T_{\alpha}} z_{\epsilon}^{-2}=2 e^{-2}(1-\beta)^{-2}\left(2 \beta-\beta^{2}\right)
$$

which surprisingly correlates very well the numerical results and has the correct asymptotic form for small values of $\beta$ except for a factor 0.99 . Fig. 16 includes the first term of the asymptotic relationship between $\theta_{1}(\infty)$ and $\Delta$ for large values of $\theta_{1}(\infty)$.

The following expression

$$
z_{\epsilon}^{2}=-\ln \left\{8 \pi \epsilon^{2} z_{\epsilon}^{2} / \beta^{2}(1-\beta)^{2}\right\}
$$

should be used in Eq. (A11) for $z_{e}$ instead of Eq. (A6), so as to include the factor $(1-\beta)^{-2}$ to account for the fact that at small values of $(1-\beta)$ the reaction zone is centered around $\chi_{r}=2 /(1-\beta)$.

However, it is clear that for small values of $(1-\beta)$ the ignition conditions result directly from the analysis of Section 5, and Eqs. (59) and (67) can be used to predict the Damköhler number of ignition with even better accuracy.

\section{Appendix B}

In the partial burning regime, the third term of the inner expansion $\Phi_{2}$ is given by the equation

$$
\begin{aligned}
d^{2} \Phi_{2} / d \zeta^{2}= & -\Lambda_{0} \exp \Phi_{1}\left[\Phi_{2}+b_{1}-\Phi_{1}{ }^{2} / T_{b}-\Phi_{1}\left(y_{F b}^{-1}+y_{0 b}^{-1}\right)\right. \\
& \left.-\zeta\left\{(\alpha+\beta) y_{0 b}^{-1}+(\beta-1) y_{F b}^{-1}-4 \pi z_{b} \exp z_{b}{ }^{2}\right\}\right]
\end{aligned}
$$

The matching conditions with the outer expansion provide the boundary conditions and an additional relation to determine the second term $b_{1}$ of the expansion for the Damköhler number. We must take into account, when writing the matching conditions, the asymptotic behaviour $\Phi_{1} \doteq-\left(T_{b}-T_{\infty}\right) \zeta / x_{b}$ for $\zeta \rightarrow \infty$, and $\Phi_{1} \doteq\left(T_{b}-T_{\infty}\right) \zeta / x_{b}+B_{1} x_{b}$ for $\zeta \rightarrow-\infty$. We thus obtain the following conditions

$$
\begin{array}{lll}
d \Phi_{2} / d \zeta=0 & \text { for } & \zeta \rightarrow \infty \\
d \Phi_{2} / d \xi=B_{1} & \text { for } & \xi \rightarrow-\infty
\end{array}
$$


A first integral of Eq. (B1), satisfying Eq. (B2) is,

$$
\begin{aligned}
\frac{d \Phi_{2}}{d \zeta} \frac{d \Phi_{1}}{d \zeta}= & -\Lambda_{0} \exp \Phi_{1}\left\{-\left(\Phi_{1}{ }^{2}-2 \Phi_{1}+2\right) T_{b}^{-1}+\Phi_{2}+b_{1}-\left(y_{0 b}^{-1}+y_{F b}^{-1}\right)\left(\Phi_{1}-1\right)\right\} \\
& +\left\{(\alpha+\beta) y_{0 b}^{-1}+(\beta-1) y_{F b}^{-1}-4 \pi z_{b} \exp z_{b}{ }^{2}\right\} \\
& \times\left\{\Lambda_{0} \zeta \exp \Phi_{1}+d \Phi_{1} / d \zeta+\left(T_{b}-T_{\infty}\right) x_{b}^{-1}\right\}
\end{aligned}
$$

and then Eq. (40) is obtained for $B_{1}$ if the boundary condition Eq. (B3) is to be satisfied.

Notice that in the second approximation the rate of decrease of temperature is not the same on both sides of the flame. Only the asymmetry of the convective effects and of the frozen flow reactant concentrations contribute to $B_{1}$.

A second integration of Eq. (B4) would be necessary if we were interested in evaluating terms of order $\epsilon^{2}$ in the expansion for the temperature and the term of order $\epsilon$ in the expansion for the Damköhler number. For the explicit determination of this term we must then find a first integral of the equation for $\Phi_{3}$.

\section{Appendix C}

We shall discuss here the asymptotic form of the solutions of Eq. (52) with the boundary conditions, Eqs. (53) and (54), for (a) small values of $m$, (b) small values of $(0.5-m)$, and $(c)$ large values of $(-m)$.

\section{(a) Solution for small $m$}

As indicated in the main text, the independent variable $\eta$ in Eq. (52) was chosen so that the factor 2 would appear in the left-hand side of the equation, otherwise, no solution of the equation would exist satisfying the boundary conditions for the particular case $m=0$. However, for $m=0$ the equation and boundary conditions are invariant under translations in $\eta$ and thus an undetermined constant appears in the solution which can be calculated if we specify that higher approximations in be bounded. This same solution is obtained by requiring that the solution for $m=0$ be the limit of the solution for small values of $m$, calculated as indicated below.

Thus for small values of $m$ Eq. (52) simplifies to

$$
2 d^{2} y / d \eta^{2}=y \exp (-y)
$$

which can be integrated once to yield

$$
(d y / d \eta)^{2}=1-(y+1) \exp (-y)
$$

if the boundary condition (53) is taken into account.

A second integration yields

$$
n-\eta=\int_{y_{0}}^{y} \frac{d u}{\sqrt{1-(u+1) \exp (-u)}}
$$

where the constant $y_{0}$ will be chosen as $y_{0}=0.9349$ so that for large values of $y$ the integral in Eq. (C3) coincides with $y$. The integrating constant $n$ appearing in Eq. 
(C3) can only be calculated if we specify that the function $y(\eta)$ given by Eq. (C3) be the first approximation $y_{1}$ in an expansion $y=y_{1}+m y_{2}$ of the solution $y$ of Eqs. (52)-(54) for small values of $m$, and that $y_{2}$ be bounded. Then we obtain

$$
n=1.344 \quad \text { and } \quad y_{1 \infty}=0
$$

for small values of $m$.

(b) Solution for $m \rightarrow 0.5$

When $m$ approaches 0.5 , both $n$ and the leakage $y_{10}$ through the reaction zone become very large compared with one. The changes in $y$ through the reaction zone remain of order unity so that in first approximation the concentration $y$ of the reactant can be taken as constant in the reaction zone $y_{1}=y_{1 \infty}$, and then the reaction zone structure coincides with that of the partial burning regime. When an analysis of Eq. (52) for small values of $(0.5-m)$, or large values of $y_{1 \infty}$, is carried out following the procedure used in Section 4 we find in first approximation

$$
y_{1 \infty}=(1-2 m)^{-1}, \quad-n=(1-2 m)^{-1}+2 \ln (1-2 m)
$$

(c) Solution for large values of $-m$

We anticipate that in the reaction region $y$ will be small, of order $1 / m$, so that Eq. (52) may be replaced in first approximation by the equation

$$
2 d^{2} y / d \eta^{2}=y \exp (-m \eta)
$$

which can be written as a Bessel equation

$$
y^{\prime \prime}+y^{\prime} / t-y=0
$$

in terms of the variable

$$
t=(-\sqrt{2} / m) \exp (-m \eta / 2)
$$

The solution of Eq. (C7), finite for $t \rightarrow \infty$,as required by the boundary condition at $\eta \rightarrow+\infty$, given by Eq. (54), is

$$
y=A K_{0}(t)
$$

where $K_{0}$ is the modified Bessel function of zeroth order, and $A$ is a constant to be determined from the boundary condition given by Eq. (53), at $\eta \rightarrow-\infty$ or $t \rightarrow 0$. For small values of $t, K_{0}$ has the expansion

$$
K_{0}=\left\{\ln (t / 2)+\gamma_{E}\right\}\left(1+t^{2} / 4 \cdots\right)+t^{2} / 4+\cdots
$$

where $\gamma_{E}=0.577$ is the Euler constant, so that we obtain, for large negative values of $\eta$, the expansion

$$
y=-\eta+\left\{2 \gamma_{E}-\ln \left(2 m^{2}\right)\right\} / m+\cdots
$$

if we choose $A=-2 / m$.

Thus

and

$$
n=\left\{2 \gamma_{E}-\ln \left(2 m^{2}\right)\right\} / m
$$

$$
y=(-2 / m) \sqrt{\pi / 2 t} \exp (-t)\left\{1-\frac{1}{8 t}+\cdots\right\}
$$


for large values of $\eta$ (and $t$ ) so that

$$
y_{1 \infty}=0
$$

\section{(d) Correlations}

The asymptotic solutions given above have been used to generate the following correlations of the results of the numerical integrations of Eqs. (52)-(54).

In the range $-0.2<m<0.5$, a good approximate expression for $n m$ is given by

$$
n m=1.344 m-4 m^{2}(1-m) /(1-2 m)+3 m^{3}-\ln \left(1-4 m^{2}\right)
$$

For small positive values of $m$ and all negative values of $m$ an approximate expression for $\mathrm{nm}$ is

$$
n m=-\ln \left(0.6307 m^{2}-1.344 m+1\right)
$$

Relative errors in these formulas do not exceed a few percent.

\section{References}

1. Spalding, D. B., Theory of Mixing and Chemical Reaction in the Opposed Jet Diffusion Flame, ARS J., 31, 763-771, (1961).

2. Liñan, A. and Da-Riva, I., Non-equilibrium Effects in Hypersonic Aerodynamics, 3rd ICAS Congress, Stockholm (1962). The Proceedings of the Congress published by Spartan Books (1964).

3. Fendell, F. E., Ignition and extinction on Combustion of Initially Unmixed Reactants, J. Fluid Mech., 21, 291-303, (1965).

4. Chung, P. M., Fendell, F. E. and Holt, J. F., Non-equilibrium Anomalies in the Development of Diffusion Flames, AIAA J., 4, 1021-1026 (1966).

5. Fendell, F. E., Flame Structure in Initially Unmixed Reactants under One Step Kinetics, Chem. Eng. Sci., 22, 1829-1837, (1967).

6. Jain, V. K. and Mukunda, H. S., On the Ignition and Extinction Problems in Forced Convection Systems, Int. Jour. Heat and Mass Transfer, 11, 491-508 (1968).

7. Jain, V. K. and Mukunda, H. S., The Extinction Problem in an Opposed Jet Diffusion Flame with Competitive Reactions, Combustion Science and Technology, 1, 105-117, (1969).

8. Sanchez Tarifa, C. Perez Del Notario, P. and Garcia Moreno, F., Combustion of Liquid Monopropellants and Bipropellants in Droplets, Eighth Symp. Int. Combustion, Williams and Wilkins, Baltimore, 1035-1053 (1962).

9. Peskin, R. L. and Wise, H., Ignition and Deflagration of Fuel Drops, AIAA J., 4, 1646-1650, (1966).

10. Peskin, R. L., Polymeropoulous, C. E. and Yeh, P. S., Results from a Theoretical Study of Fuel Drop Ignition and Extinction, AIAA J., 5, 2173-2178, (1967).

11. Kassoy, D. R., and Williams, F. A., Effects of Chemical Kinetics on Near Equilibrium Combustion in Nonpremixed Systems, Phys. of Fluids, 11, 1343-1351, (1968).

12. Kassoy, D. R., Liu, M. K. and Williams, F. A., Comments on Effects of Chemical Kinetics on Near-equilibrium Combustion in Non-premixed Systems, Phys. of Fluids, 12, 265-267, (1969).

13. Zeldovich, Y. B., On the Theory of Combustion of Initially Unmixed Gases, NACA Tech. Mem., No 1296, (1951).

14. Friedlander, S. K. and Keller, K. H., The Structure of the Zone of Diffusion-Controlled Chemical Reaction, Chem. Eng. Sci., 18, 365, (1963).

15. Kirkby, L. L. and Schmitz, R. A., An Analytical Study of the Stability of a Laminar Diffusion Flame, Combustion and Flame, 10, 205-220, (1966).

16. Schmitz, R. A., A Further Study of Diffusion Flame Stability, Combustion and Flame, 11, 49-62, (1967). 
17. Frank-Kamenetskii, Diffusion and Heat Transfer in Chemical Kinetics, Chapter VI., Pergamon Press, (1969).

18. Burke, S. P. and Schumann, T. E. W., Diffusion Flames, Ind. and Eng. Chemistry, 20, 998-1004, (1928).

19. Williams, F. A., Combustion Theory, Addison Wesley, (1965).

20. Librovitch, V. B., Personal communication, (1970).

21. Liñan, A. On the Internal Structure of Laminar Diffusion Flames, OSR/EOAR, TN 62-69. INTA, Madrid, (1961).

22. Liñan, A., On the Structure of Laminar Diffusion Flames, Tech. Rept. FM 63-2. INTA, Madrid, (1963).

23. Liñan, A., Diffusion Flames and Supersonic Combustion, Annu. Sci. Rept. No 1. EOAR Cont. No F61052-69-C-0036. INTA, Madrid (1970).

24. Libby, P. A., and Economos, E., A Flame Zone Model for Chemical Reaction in a Laminar Boundary Layer with Applications to the Injection of Hydrogen-Oxygen Mixtures, Int. J. Heat Mass Transfer, 6, 113-128 (1963).

25. Liñan, A., and Crespo, A., An Asymptotic Analysis of Unsteady Diffusion Flames for Large Activation Energies, Tech. Rep. for ARO-INTA Subcontract, Madrid (1972). 